\title{
Thermodynamic Analysis and Algorithm Optimisation of a Multi-stage Compression Adiabatic Compressed Air Energy Storage System
}

\author{
Jie Song ${ }^{1}$, Xiaodong Peng ${ }^{1}$, Xiangjun Fang ${ }^{2}$, Ying $\operatorname{Han}^{2}$, Zhanfeng Deng ${ }^{1}$, Guizhi Xu ${ }^{1}$, \\ Lixiao Liang ${ }^{1}$, Jibiao Hou ${ }^{1}$, Hongwei $\mathrm{Wu}^{3 *}$ \\ ${ }^{1}$ State Key Laboratory of Advanced Power Transmission Technology \\ (Global Energy Interconnection Research Institute, Changping District, Beijing, 102211, China) \\ ${ }^{2}$ School of Energy and Power Engineering, Beihang University, Beijing, 100191, China \\ ${ }^{3}$ School of Engineering and Computer Science, University of Hertfordshire, Hatfield, AL10 9AB, UK
}

*Corresponding author: Dr. Hongwei Wu, Email: h.wu6@herts.ac.uk

\begin{abstract}
:
In this article, a novel multi-stage compression and heat recovery on an adiabatic compressed air energy storage (A-CAES) system is proposed. In the current work, an in-house code named CAESSC 1.0 is successfully developed which can be helpful to evaluate the performance of the proposed ACAES system and other power generation systems. In order to obtain the optimum performance, thermodynamic analysis of a multi-stage compression A-CAES system is investigated in a systematic manner. The effect of several control parameters, such as gas storage methods, storage pressures, interstage heat transfer methods, and stage numbers of the compressor as well as the turbine on the overall performance of the system is discussed in detail. Results indicate that using constant pressure gas storage method could significantly improve both the energy storage efficiency and the energy storage density of the system. An optimised algorithm of the heat exchanger in CAES system is proposed to remarkably improve the simulation performance. The highest efficiency can exceed $70 \%$ when using compressed air with adiabatic method. Two different gas storage methods, i.e. constant volume and constant pressure, have been discussed. It indicates that the efficiency of the system under constant pressure storage is about $4 \%$ higher than that under constant volume storage.
\end{abstract}

Keywords : thermodynamic analysis; adiabatic compressed air energy storage; heat recovery; energy efficiency. 


\begin{tabular}{|c|c|c|c|}
\hline \multicolumn{2}{|c|}{ Nomenclature } & \multicolumn{2}{|l|}{ Subscripts } \\
\hline$A$ & Area $\left[\mathrm{m}^{2}\right]$ & $a m b$ & Ambient \\
\hline$A, \ldots, N$ & Polynomial coefficient [-] & co & Compressor \\
\hline$C_{p}$ & $\begin{array}{l}\text { Specific heat at constant pressure } \\
{[\mathrm{kJ} /(\mathrm{kg} \cdot \mathrm{K})]}\end{array}$ & cool & Cooling or low temperature \\
\hline$e_{1}$ & Available energy $[\mathrm{kJ}]$ & elc & Other electricity consumption \\
\hline$e_{L}$ & Available energy loss $[\mathrm{kJ}]$ & enc & Other energy consumption \\
\hline$F$ & Force $[N]$ & $g e$ & Generator \\
\hline$G$ & $\begin{array}{l}\text { System operating mass flow rate } \\
{[\mathrm{kg} / \mathrm{s}]}\end{array}$ & hot & Hot or high temperature \\
\hline$g$ & Acceleration of gravity $\left[\mathrm{km} / \mathrm{s}^{2}\right]$ & $i$ & Order \\
\hline$h$ & enthalpy $[\mathrm{kJ}]$ & in & Inlet/input \\
\hline$\Delta h$ & enthalpy difference $[\mathrm{kJ}]$ & $L$ & Loss \\
\hline$k$ & Adiabatic index of air [-] & $\max$ & Maximum \\
\hline$L$ & Length $[\mathrm{m}]$ & & \\
\hline$m$ & Mass flow rate $[\mathrm{kg} / \mathrm{s}]$ & me & Motor \\
\hline $\mathrm{n}$ & $\begin{array}{l}\text { Number of stages of the compressor } \\
\text { /turbine [-] }\end{array}$ & $\min$ & Minimum \\
\hline$\Delta p$ & pressure head $[\mathrm{kPa}]$ & out & Outlet/output \\
\hline $\mathrm{P}$ & Power $[\mathrm{kW}]$ & sto & Storage \\
\hline$P$ & Pressure $[\mathrm{kPa}]$ & & \\
\hline$q$ & Energy flow $[\mathrm{kJ} / \mathrm{kg}]$ & & \\
\hline$Q$ & Energy/enthalpy $[\mathrm{kJ}]$ & \multicolumn{2}{|c|}{ abbreviation } \\
\hline$R$ & Gas constant $[\mathrm{J} /(\mathrm{mol} \cdot \mathrm{K})]$ & CAES & Compressed air energy storage \\
\hline$T$ & Temperature $[\mathrm{K}]$ & CHTFST & Cool heat transfer fluid storage tank \\
\hline$v$ & Velocity [V] & $\mathrm{HE}$ & Heat exchanger \\
\hline $\mathrm{V}$ & Volume $\left[\mathrm{m}^{3}\right]$ & HHTFST & Hot heat transfer fluid storage tank \\
\hline \multirow[t]{2}{*}{$W$} & Work $[\mathrm{kJ}]$ & HPC & High pressure compressor \\
\hline & & HPT & High pressure turbine \\
\hline \multicolumn{2}{|c|}{ Greek symbols } & HTF & Heat transfer fluid \\
\hline$\eta$ & Efficiency [-] & LPC & Low pressure compressor \\
\hline$\pi$ & Ratio [-] & LPT & Low pressure turbine \\
\hline$\rho$ & Density $\left[\mathrm{kg} / \mathrm{m}^{3}\right]$ & REG & Renewable energy generation \\
\hline$\sigma$ & Pressure recovery coefficient [-] & TPR & Throttling pressure ratio \\
\hline$\Phi$ & Heat flow $[\mathrm{kJ} / \mathrm{s}]$ & & \\
\hline
\end{tabular}




\section{Introduction}

Over the past few decades, global electricity production increased steadily. It is noticed that by the end of 2017 the global electricity production has reached 25,592 TWh. It is also recognized that with the rapid development of renewable energy, such as hydro power, solar power and wind power, the proportion of renewable energy achieves around $24.8 \%$ in all energy sources in 2017. It should be stressed that, however, renewable energy cannot replace all traditional fossil fuels due to the intrinsic intermittence and fluctuation. It is known that electrical energy storage (EES) plays an important role in balancing energy production and demand in order to solve the intermittent issue of wind and solar energy sources [1-2]. Nowadays, several EES technologies, i.e. CAES, thermal energy storage (TES), pumped hydroelectric (PHS), batteries, flywheel, capacitor have been widely developed. In 2015, Luo et al. [2] performed a detailed and critical review to evaluate both the advantages and disadvantages of the above technologies in terms of energy efficiency and density, power range, investment cost and time of response.

Among EES technologies, the advantages of long lifetime, high reliability and economic competitiveness of a CAES make it successfully apply in storing large-scale energy (tens or hundreds of MW) [3,4]. In a traditional CAES power plant, a multi-stage compressor will compress the ambient air with intercooling in order to increase the compression efficiency and the energy density during the energy charging period. Afterwards, it will be stored in an underground reservoir, which is normally the saline dome caverns, porous rock formation or old mines [5]. During the energy discharging period, prior to expanding into the gas turbine for power generation, the natural gas will be burned to heat the compressed air. In 1978, the first commercial CAES plant, the Huntorf power station with a storage power capacity of $60 \mathrm{MW}$ and a discharge power of $290 \mathrm{MW}$ was put into operation [6]. Later on, in 1991, the second commercial CAES plant, the Mcintosh power station with storage power capacity of $50 \mathrm{MW}$ and discharge power of $110 \mathrm{MW}$ was successfully developed and applied [7]. In 2001, a large CAES power station was built in Ohio, it consisted of nine $300 \mathrm{MW}$ units with a total power of $2700 \mathrm{MW}$ [8].

Over the past two decades, CAES technology has attracted wide attention and many research have been devoted to CAES. Guo et al. developed a theoretical thermodynamic model for an A-CAES system in order to investigate the effect of control parameters such as air temperature and pressure on both energy density and roundtrip efficiency [9]. It was found that the design capacity of the first heat exchanger of the expansion can be affected by the dynamic characteristics of the compressed air storage system. It can also reduce the roundtrip efficiency and energy density. He et al. presented a methodology which can be used to calculate the total exergy of a predefined storage volume. The proposed methodology was validated against test data of the Huntorf plant [10]. In their study, two cavern operation scenarios, i.e. uncompensated and compensated isobaric cavern with three different wall heat transfer coefficients, were investigated. Within the operating conditions of the Huntorf plant, their results indicated that $34.77 \%$ more exergy after charging and $37.98 \%$ more exergy after throttling can be stored in the cavern compared to those in the cavern with adiabatic condition. Most recently, Mohamad et al. developed a steady state thermodynamic analysis on a concept of trigenerative compressed air energy storage system (T-CAES) and validated against experimental data [11] during the charge, storage and discharge stages. It was concluded that by adding the cooling and heating potentials, the system efficiency could be increased remarkably. Zhang et al. developed a theoretical model under steady state operating condition, sensitivity analyses were conducted in a systematic manner [12]. It was found that the total exergy destruction coefficient could be determined by the heat transfer coefficient, injected air temperature, and initial temperature of the cavern. Al Jubori and Jawad carried out a combined mean-line design and three dimensional (3-D) numerical simulation to investigate the performance of a radial-inflow expander for a small scale CAES system [13]. Their results showed that the radial-inflow expander with CAES cold be used for distributed power generation. Guo et al., developed a CAES with low-temperature thermal energy storage (TES) to study the effects of several control parameters, such as component thermal inertia, volumetric effects of pipes in overall energy storage process and start-up stage [14]. Their results showed that, 
during the discharging process, more energy loss $(4.17 \%)$ from heat exchangers of compressors is observed compared to that with heat exchangers of expanders $(2.5 \%)$.

Most recently, it is interesting to note that several research works focused on advanced adiabatic compressed air energy storage (AA-CAES) power station [15]. AA-CAES has received many attractions since it has potential of large-scale energy storage and high energy recovery. It is recognized that for a traditional CAES system, system efficiency is low, which is around 30\% - 43\%; whereas for AA-CAES system, the efficiency is approximately 50\% - 75\% [16-18]. Mozayeni et al. performed a thermodynamic analysis to systematically evaluate the effect of storage pressure, pre-set pressure along with compressor and turbine efficiencies on the overall AA-CAES system performance [19]. It was found that the amount of energy stored in an AA-CAES system and power generated by the expander strongly depends on the storage pressure. In the research work of Geissbuhler et al. [20, 21], several different operating conditions were investigated for an AA-CAES pilot-scale plant, such as charging/discharging cycles of various durations with air temperatures of up to $550{ }^{\circ} \mathrm{C}$ and maximum cavern gauge pressure of 7 bar $[20,21]$. Han and Guo applied a threeoperation-mode of expanders such as constant pressure, constant-sliding and sliding pressure model to investigate the discharge characteristics of an AA-CAES system [22]. Their research work may improve the overall performance for a tri-generative system. Besides AA-CAES, many efforts have been made on other types of CAES. Yang et al. carried out daily cycle, weekly cycle and monthly cycle to evaluate the CAES performance within a month working cycle. They stated that the energy recovery efficiencies of $96.96 \%, 96.27 \%$ and $93.15 \%$ could be achieved respectively for each cycle times [23]. Meng et al. proposed an integrated CAES system with a solar transcritical carbon dioxide Rankine cycle especially focusing on negative impacts of the pump and heat recovery. Their results illustrated that the negative effect of the pump power on the system performance become severe at high mass flow rate. Moreover, compression heat recovery results in a low exergy efficiency at initial charging process [24]. Mohamad et al. proposed a Micro-scale trigenerative CAES system based on existing technologies. However, due to exergy loss in the throttling valve, the electrical efficiency was achieved with a low value of $17 \%$, while the comprehensive efficiency could reach $27.2 \%$ [25]. Chen et al. proposed a modelling methodology for developing the model of a compressed air energy storage system. It was found that the efficiency of the designed experimental setup in differenct conditions ranges from $13 \%$ to $25 \%$, and the optimal system efficiency will increase to $60 \%$ for a triple-stage system [26].

A-CAES system presents a relative higher exergy efficiency during the heat exchanging process. However, it appears from the previous investigation that there were less reports on A-CAES system. It is imperative to further develop a thermodynamic process of A-CAES and there is still much room to be improved in this area. A thermodynamic analysis of a multi-stage compression A-CAES system is investigated to improve the system performance. The objective of the current work is to propose a novel A-CAES system and to explore the physical phenomena pertinent, as well as to validate and test the designed in-house code. In this study, the effect of several control parameters, such as gas storage methods, storage pressures, interstage heat transfer methods, and multi-stages of the compressor and the turbine on the overall thermal performance will be discussed. Meanwhile, a novel internal temperature solution arithmetic is proposed and it can be applied to all occasions involving heat exchanger design and solution, thereby enhancing the system efficiency accurately and intelligently.

\section{System description}

\subsection{System configuration}

Fig. 1 depicts the schematic drawing of the proposed A-CAES system. The proposed system mainly consists of multistage compressor with intercoolers, multistage expander, cold storage/heat exchanger, heat storage/heat exchanger, storage tank, motor, and generator. The constant pressure operation mode will be taken into accout in this study and the working principle is presented as follows:

(1) Energy storage process 
The off-peak power, also known as surplus electricity, will be used to drive the compressor, leading air to a high pressure, then compression heat and compressed air are generated. The compression heat will be transferred into the heat transfer fluid (HTF) through the heat exchangers (HEX) and stored in the hot heat transfer fluid storage tank (HHTFST). While the compressed air will be stored into an underground cavern, which is filled with water in order to maintain a constant pressure.

(2) Energy release process

At peak hours, the hot HTF in HHTFST will be used for heating the compressed air at turbine inlet, then HTF flows into the cool heat transfer fluid storage tank (CHTFST). Afterwards, the hightemperature compressed air will flow into the turbine to drive the generator to produce power if needed. Finally, exhausting air will be released to the environment.

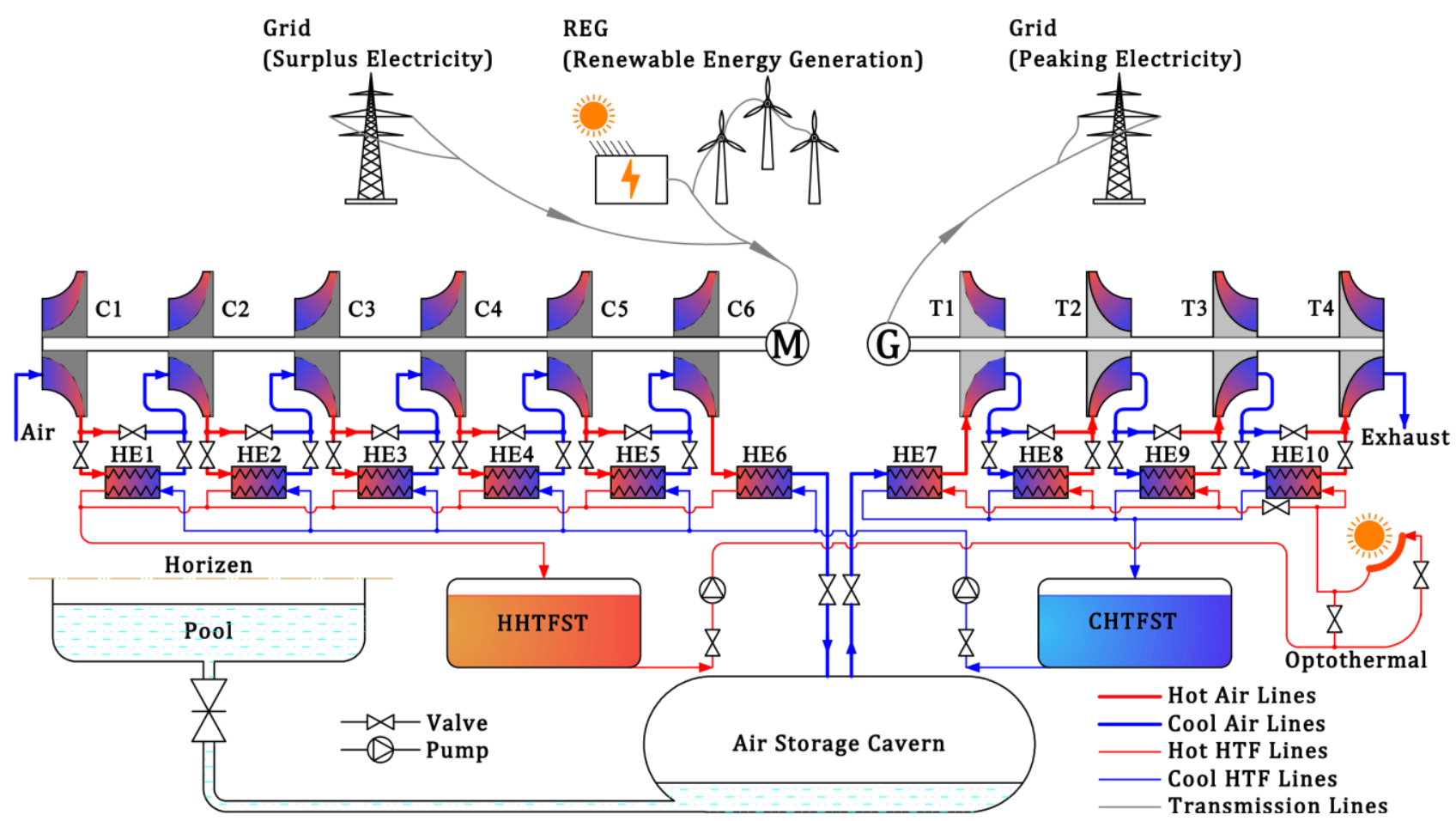

Fig. 1. Overall schematic of compressed air energy storage system.

\subsection{System component modelling}

To evaluate the performance of the proposed A-CAES system, several assumptions are made as follows:

- The system is in a steady state.

- There are no heat losses through pipelines and each component.

- The air after purification consists of $78.12 \%$ nitrogen, $20.96 \%$ oxygen and $0.92 \%$ argon.

- Isentropic efficiency model is used to evaluate the power generation or consumption by the compressor, turbine and the pump.

- Heat transfer in heat exchangers complies with energy conservation and pinch point limitation.

In order to maximize the use of compression heat, in the current work, it is proposed to use compressed air as the heat transfer medium to replace the heat transfer oil and other media, which can improve the utilization rate of the compression heat and thus improve the efficiency of the overall energy storage system.

\subsubsection{Energy storage process}

Air compressor is one of the key components at energy storage stage. The centrifugal compressor is set with a pressure ratio of 2, thus, a multi-stage compression is required to compress the air. After first-stage compression, air outlet temperature will be greater than $100^{\circ} \mathrm{C}$. Air temperature will exceed 
the required value without applying interstage cooling, the compressor therefore cannot work properly. It is important to cool down the air in order to achieve a higher system efficiency, the recovered heat can be reused to interstage heating in the expander later when power is needed. The compressor model in the current work can be described as follow [20] :

$$
\left\{\begin{array}{l}
T_{\text {out } \_c o}=T_{i n_{-} c o}+W_{c o} /\left(C_{p} m_{c o}\right) \\
W_{c o}=\frac{1}{\eta_{c o}} m_{c o} \frac{k}{k-1} R T_{i n_{-} c o}\left[\left(\pi_{c o}\right)^{\frac{k-1}{k}}-1\right] \\
p_{\text {out_co }}=\pi_{c o} p_{i n_{-} c o} \\
\eta_{c o}=\left[\left(-2.2 \pi^{2}+9.7 \pi+73.0\right)\left(-0.08972 G_{c o r}{ }^{2}+1.3873 G_{c o r}+78.7\right)\right]^{0.5} / 100 \\
G_{c o r}=G \frac{101325}{P_{i n_{-} c o}} \sqrt{\frac{T_{i n_{-} c o}}{288.15}}
\end{array}\right.
$$

where $C_{p}$ is the specific heat at constant pressure, taking air $C_{p}=1.004, k$ is the adiabatic exponent or heat capacity ratio, taking air $\mathrm{k}=1.4, R$ is gas constant, $W_{c o}$ is the compressor work, $\eta_{c o}$ is adiabatic efficiency of compressor, $m_{c o}$ is compressor mass flow rate, $\pi_{c o}$ is pressure ratio of compressor, $T_{i n \_c o}$ and $T_{\text {out_co }}$ are inlet and outlet temperature of each compressor stage, $p_{\text {in_co }}$, and $p_{\text {out_co }}$ are inlet and outlet pressure of each compressor stage, $G$ and $G_{c o r}$ are operating mass flow rate and application range of flow rate.

At the compression process, the calculation of energy consumption under three different compression methods can be described as:

Isothermal compression:

$$
W_{c o \_i s o}=R T_{c o \_i n} \ln \pi_{c o}
$$

Adiabatic compression:

$$
W_{c o \_a d i}=\frac{k}{k-1} R T_{c o_{-} i n}\left(\pi_{c o}^{\frac{k}{k-1}}-1\right)
$$

Polytropic compression - multistage compression with intercooling (assuming that the inlet total temperature and compression ratio of each stage are equal):

$$
W_{c o_{-} p o l}=\frac{k}{k-1} n R T_{c o_{-} i n}\left(\pi_{c o}^{\frac{k-1}{n k}}-1\right)
$$

Based on the above three methods, the energy consumption profile of three different compression processes can be demonstrated in Fig. 2. Under the current conditions in Fig. 2, it can be seen clearly that the compression input power is proportional to the exhaust pressure. Under the same exhaust pressure, adiabatic compression case shows the highest compression input power, whereas the isothermal compression process shows the lowest. It could be concluded that the multistage compression process with intercooling can be regarded as an isothermal compression process. 


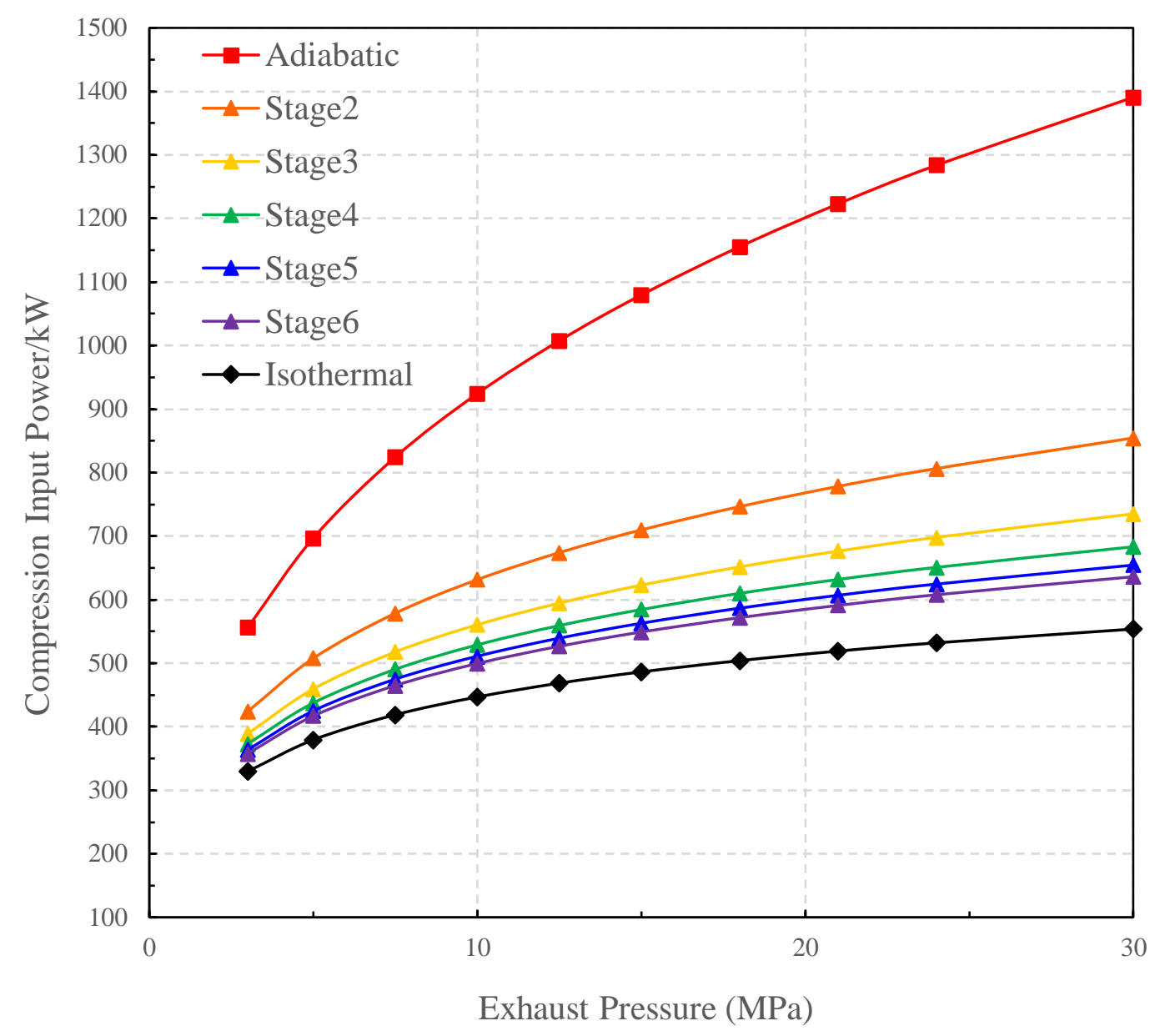

Fig. 2. Energy consumption surface diagram of the different compression processes.

It should be noted that the designed compression ratio and efficiency of each stage may not be equal. For the sake of simplicity, a constant total compression ratio is assumed, and the distribution principles of each stage's compression ratio are as follows:

(1) to obtain the minimum total power consumption of multistage compressor;

(2) to make each impeller to work at high efficiency at the same time.

The total power consumption for unit mass gas can be represented as:

$$
\begin{aligned}
& W_{c o}=\sum_{i=1}^{N} W_{c o_{-} i}=W_{c o_{-} 1}+W_{c o_{-} 2}+W_{c c_{-} 3}+W_{c o_{-} 4} \\
& =\frac{k}{k-1} R\left[\frac{T_{c c_{-} i n_{-} 1}}{\eta_{c o_{-} 1}}\left(\pi_{c c_{-} 1}^{\frac{k-1}{k}}-1\right)+\frac{T_{c o_{-} i n_{-} 2}}{\eta_{c o_{-} 2}}\left(\pi_{c c_{-} 2}^{\frac{k-1}{k}}-1\right)+\frac{T_{c o_{-} i n_{-} 3}}{\eta_{c c_{-} 3}}\left(\pi_{c o_{-} 3}^{\frac{k-1}{k}}-1\right)+\frac{T_{c o_{-} i n_{-} 4}}{\eta_{c o_{-} 4}}\left(\pi_{c o_{-}-4}^{\frac{k-1}{k}}-1\right)\right]
\end{aligned}
$$

Ignore the influence of pressure ratio on efficiency, let $Z_{i}=\frac{T_{c o_{-} i n_{-} i}}{\eta_{c o_{-} i}}$, then Eq. (5) can be simplified as follows:

$$
W_{c o}=\frac{k}{k-1} R\left[Z_{1}\left(\pi_{c o_{-}-1}^{\frac{k-1}{k}}-1\right)+Z_{2}\left(\pi_{c o_{-} 2}^{\frac{k-1}{k}}-1\right)+Z_{3}\left(\pi_{c o_{-}}^{\frac{k-1}{k}}-1\right)+Z_{4}\left(\pi_{c o_{-}-1}^{\frac{k-1}{k}}-1\right)\right]
$$

where, $Z, R$ and $k$ are constant, according to the average inequality, the following inequality can be obtained: 


$$
\begin{aligned}
& W_{c o}=\frac{k}{k-1} R\left[Z_{1}\left(\pi_{c o_{-} 1}^{\frac{k-1}{k}}-1\right)+Z_{2}\left(\pi_{c o_{-} 2}^{\frac{k-1}{k}}-1\right)+Z_{3}\left(\pi_{c o_{-} 3}^{\frac{k-1}{k}}-1\right)+Z_{4}\left(\pi_{c o_{-} 4}^{\frac{k-1}{k}}-1\right)-\left(Z_{1}+Z_{2}+Z_{3}+Z_{4}\right)\right] \\
& \geq \frac{k}{k-1} R\left[4 \sqrt[4]{Z_{1} Z_{2} Z_{3} Z_{4}\left(\pi_{c o_{-} 1} \pi_{c o_{-} 2} \pi_{c o_{-} 3} \pi_{c o_{-} 4}\right)^{\frac{k-1}{k}}}-\left(Z_{1}+Z_{2}+Z_{3}+Z_{4}\right)\right]
\end{aligned}
$$

when $Z_{1} \pi_{c o_{-} 1}^{\frac{k-1}{k}}=Z_{2} \pi_{c o_{-} 2}^{\frac{k-1}{k}}=Z_{3} \pi_{c o_{3} 3}^{\frac{k-1}{k}}=Z_{4} \pi_{c o_{-} 4}^{\frac{k-1}{k}}$, then the minimum value of $W_{c o}$ can be achieved. Define the total pressure loss coefficients of the four heat exchangers are $\sigma_{i}$, the total compression ratio is $\pi_{c o}$, then $\pi_{c o}=\pi_{c o_{-}-} \pi_{c o_{-} 2} \pi_{c o_{-} 3} \pi_{c o_{-} 4} \sigma_{1} \sigma_{2} \sigma_{3} \sigma_{4}$. As a consequence, the optimal compression ratio distribution can be obtained as:

$$
\pi_{c o_{-} 1}=\frac{\sqrt[4]{\frac{\pi_{c o}}{\sigma_{1} \sigma_{2} \sigma_{3} \sigma_{4}}\left(Z_{1} Z_{2} Z_{3} Z_{4}\right)^{\frac{k-1}{k}}}}{Z_{1}^{\frac{k-1}{k}}}, \pi_{c o_{-} i}=\pi_{c o_{-} 1}\left(\frac{Z_{1}}{Z_{i}}\right)^{\frac{k-1}{k}},(i=2,3,4)
$$

The energy consumption of multistage compression approaches the minimum at the equal compression ratio distribution. The adiabatic index $k$ in Eq. (8) can be a variable, and the compression model for ideal air cannot be applied for real air. Thus, the enthalpy difference method is normally used to solve actual air compression process. The specific solution steps are illustrated in Fig. 3.

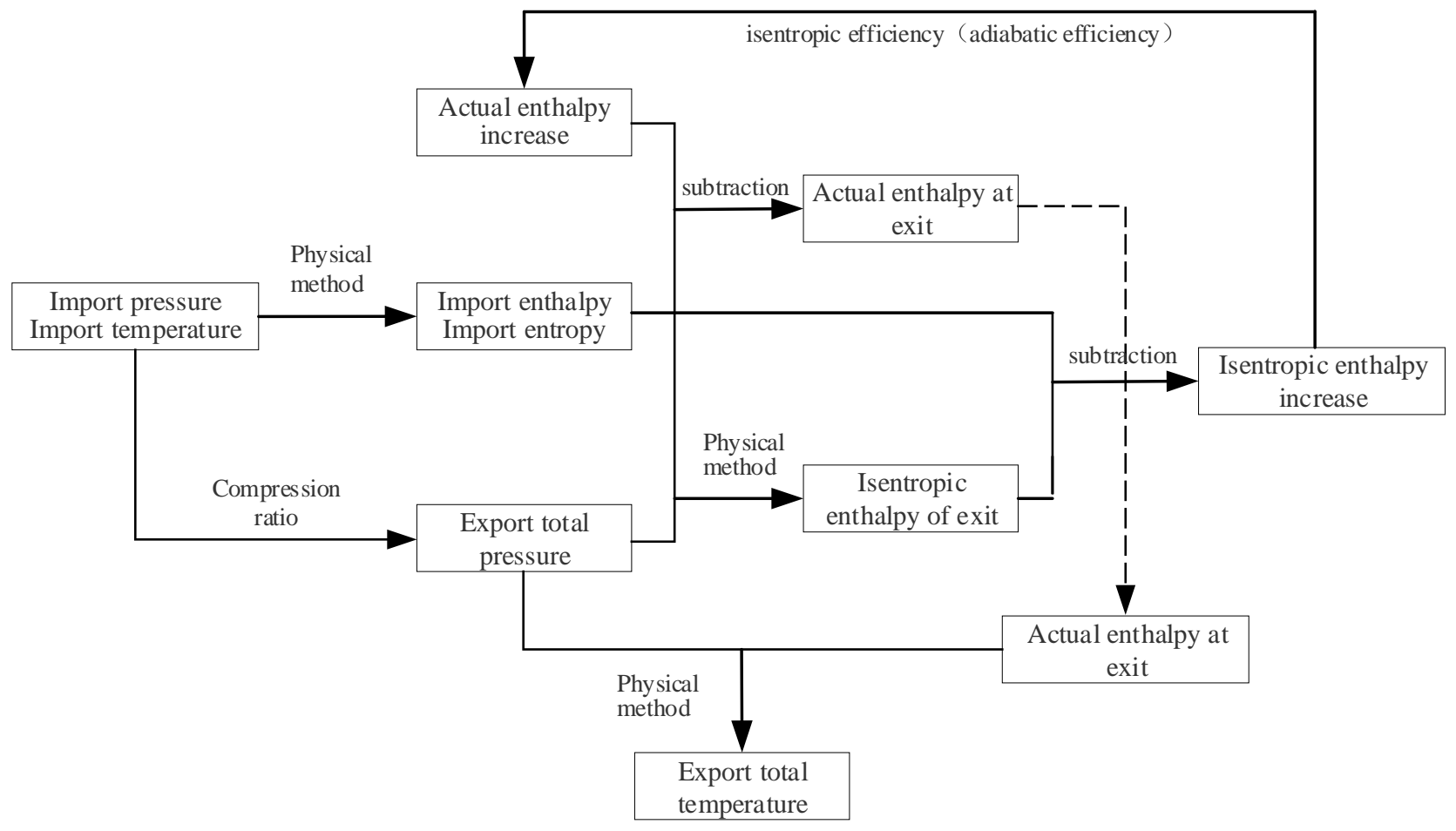

Fig. 3. Flow diagram of compressor solution model

\subsubsection{Energy release process}

The multi-stage expansion needs to be applied when fully releasing the compressed air. After each stage of expansion, the compressed air will be reheated before entering the next stage of expansion. Therefore, the mechanical work of expansion will increase and the efficiency of the overall expansion power machine will be further improved. The expander model can be described as: 


$$
\left\{\begin{array}{l}
T_{\text {out_ex }}=T_{i n_{-} e x}-w_{e x} /\left(C_{p} m_{e x}\right) \\
W_{e x}=\eta_{e x} m_{e x} \frac{k}{k-1} R T_{i n_{-} e x}\left[1-\left(\frac{1}{\pi_{e x}}\right)^{\frac{k-1}{k}}\right] \\
p_{\text {out_ex }}=\left(1 / \pi_{e x}\right) p_{\text {in_ex }} \\
\eta_{\text {ex }}=\left[\left(-0.5073 \pi^{2}+2.8188 \pi+81.95\right)\left(-0.04959 G_{c o r}{ }^{2}+0.6446 G_{c o r}+83.6\right)\right]^{0.5} / 100 \\
G_{\text {cor }}=G \frac{101325}{P_{\text {in_ex }}} \sqrt{\frac{T_{\text {in_ex }}}{288.15}}
\end{array}\right.
$$

where $W_{e x}$ is the expander does work per stage, $\eta_{e x}$ is adiabatic efficiency of expander, $m_{e x}$ is mass flow rate of expander, $\pi_{e x}$ is pressure ratio of expander, $T_{\text {in_ex }}$ and $T_{\text {out_ex }}$ are inlet and outlet temperature of expander, $p_{\text {in_ex }}$, and $p_{\text {out_ex }}$ are inlet and outlet pressure of each stage expander.

During the energy release process, CAES system will adopt a series of multistage units for air expansion. However, their working principle is different. The expressions of energy output under three different expansion methods are as follows:

Isothermal expansion:

$$
W_{e x_{-} i s o}=R T_{e x_{-} i n} \ln \pi_{e x}
$$

Adiabatic expansion:

$$
W_{\text {ex_adi }}=\frac{k}{k-1} R T_{e x_{-} i n}\left(1-\pi_{e x}^{\frac{1-k}{k}}\right)
$$

Polytropic expansion - multistage expansion with reheating between stages:

$$
W_{e x_{-} p o l}=\frac{k}{k-1} n R T_{e x_{-} i n}\left(1-\pi_{e x}^{\frac{1-k}{n k}}\right)
$$

The expansion ratio distribution principles of multistage turbines are similar to that in compressor. As is always the case, reheating, compression heat, solar heat or fuel combustion heat can be used to improve the inlet gas temperature of turbines. Fig. 4 shows one-dimensional solution for a single stage turbine or one stage of a multi-stage turbine, the air is heated before every expansion process. However, since the hot oil extracts the thermal energy from compressed air, the heat capacity is limited, thus the thermal energy cannot afford the energy requirements by unlimited multi-stage expansion. Suitable stage number which would match the heat capacity needs to be determined to make the system works efficiently. 


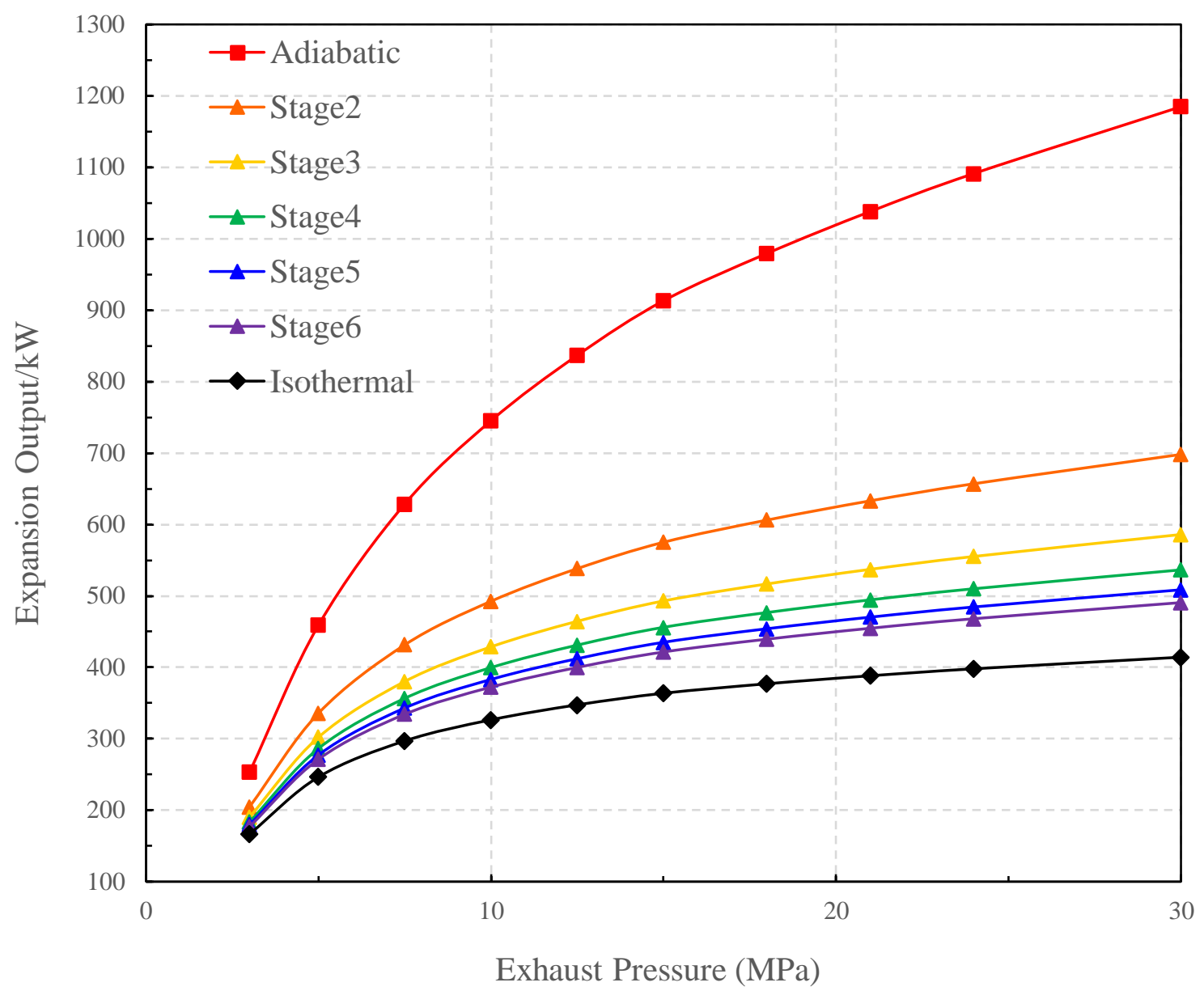

Fig. 4. Energy release surface diagram of the different expand processes.

Similarly, the enthalpy difference solution model of the actual air expansion machine can be illustrated in Fig. 5.

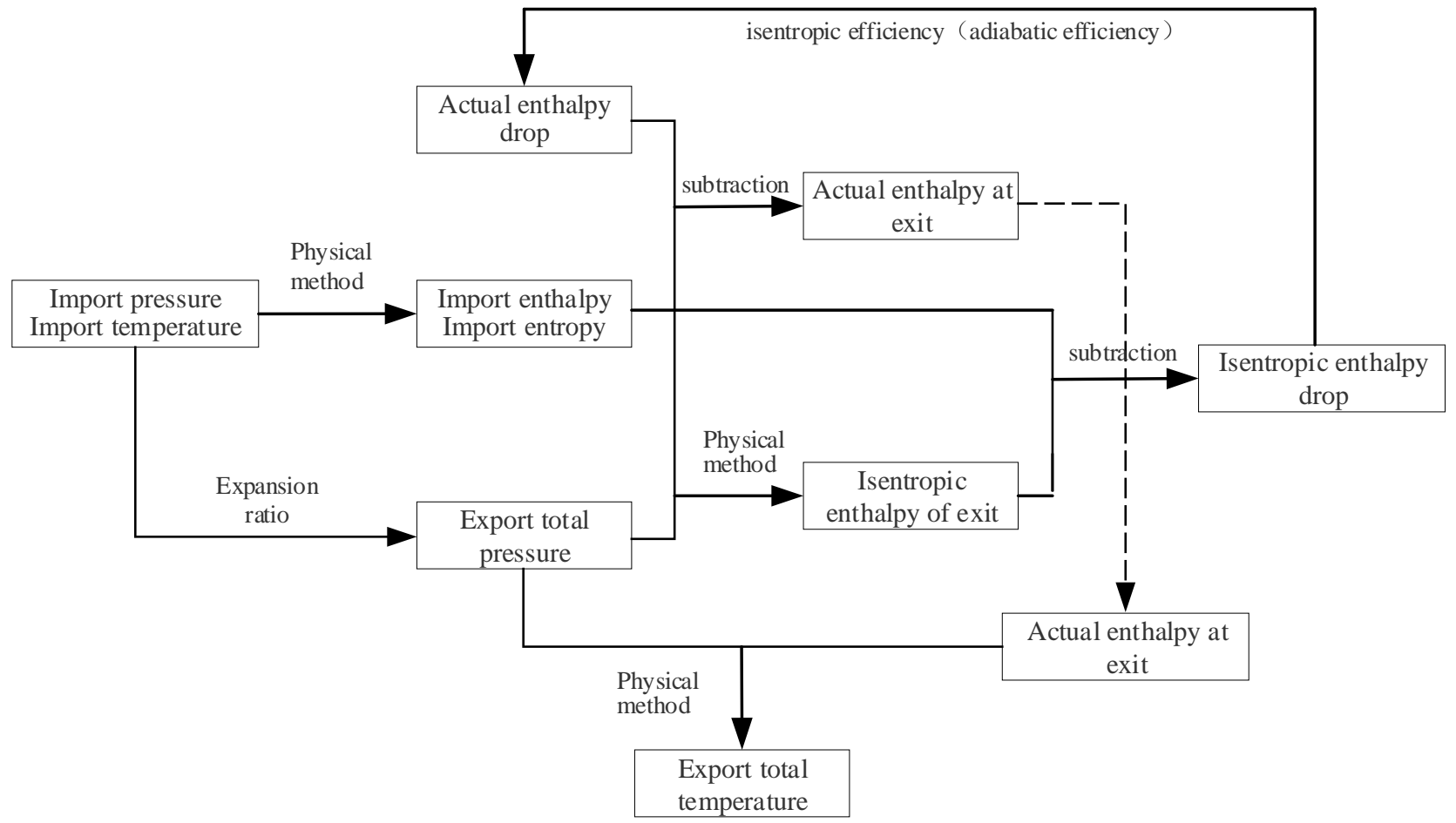

Fig. 5. Expansion machine solving model. 


\subsubsection{Pump solution model}

In an A-CAES system, pump components mainly include liquefied air booster pump (cryogenic pump) and heat transfer oil, lubricating oil delivery pump, air compressor with auxiliary watercooling pumps. As a supercharging device, although the working medium is liquid, the pump machine can be solved using enthalpy difference model. In addition, the unique solution method of pump machinery can be adopted, and the power can be solved by the following equivalent formulas.

$$
\mathrm{P}=F v=\Delta p m / \rho=m g H=\rho V g H
$$

where $F$ is force acting in the direction of the liquid under pressure, $v$ is velocity in the direction of motion of the liquid under pressure, $\Delta p$ is pressure head, $A$ is area under force in the direction of movement, $\rho$ is density of liquid medium, $g$ is acceleration of gravity.

Through the comparison, it is found that the difference of pump power obtained by the two methods is less than $1 \%$. The former (enthalpy difference method) is used for high pressure pump, while the latter is used for low pressure pump, both with high accuracy.

\subsubsection{Heat exchanger model}

The heat exchanger mainly includes two aspects: one is to use heat (enthalpy) exchange to determine the unknown temperature through the known one; while the second is to strictly control the minimum temperature difference of the heat flow during the overall heat transfer process to meet the requirements of heat exchanger.

\subsubsection{Heat (enthalpy) exchange algorithm}

The heat added by the isobaric process of ideal gas is as follows:

$$
q=C_{p}\left(T_{\text {out }}-T_{\text {in }}\right)=h_{\text {out }}-h_{\text {in }}
$$

The work done by expanders at each stage is enthalpy difference between inlet and outlet. For the case of sufficient heat, air temperature in the heater drops from $T_{\text {in }}$ to $T_{\text {out }}$, then rises from $T_{\text {out }}$ to $T_{\text {in }}$, where the temperature difference is assumed to be the same. For ideal gas with constant specific heat, the enthalpy difference is also the same. Therefore, from theoretical point of view, it can be considered that the heat $q$ absorbed by the gas in the turbine front heaters at all stages is equal to the work done by the turbine expander.

Efficiency formula of heat exchanger is:

$$
\begin{gathered}
\eta=\frac{\phi}{\phi_{\max }}=\frac{W_{1}\left(T_{i n_{-} 1}-T_{\text {out } 1}\right)}{W_{\min }\left(T_{i n_{-} 1}-T_{i n_{-} 2}\right)}=\frac{W_{2}\left(T_{\text {out } 2}-T_{i n_{-} 2}\right)}{W_{\min }\left(T_{i n_{-} 1}-T_{i n_{-} 2}\right)} \\
W=C_{p} m
\end{gathered}
$$

where $\eta$ is the heat exchanger efficiency, $\Phi, \Phi_{\max }$ are actual heat flow of heat exchanger and its maximum value, $T_{\text {in_l }_{-}, T_{\text {out }} 1}$ are medium 1 inflow and outflow temperature, and $T_{\text {in_2 }}, T_{\text {out_ } 2}$ are medium 2 inflow and outflow temperature, if $W_{1}=W_{2}=W_{\min }$, formula (3) is simplified to:

$$
\eta=\frac{T_{i n_{-} 1}-T_{\text {out_1 }}}{T_{i n_{-} 1}-T_{i n_{-} 2}}=\frac{T_{\text {out } 2}-T_{i n_{-} 2}}{T_{i n_{-} 1}-T_{i n_{-} 2}}
$$

From the above, the heat exchanger model is:

$$
\left\{\begin{aligned}
T_{\text {out } \_2} & =\eta T_{i n_{-} 1}+(1-\eta) T_{i n_{-} 2} \\
T_{\text {out } 11} & =\eta T_{i n_{-} 2}+(1-\eta) T_{i n_{-} 1} \\
Q & =m\left(h_{\text {out_2 }}-h_{i n_{-} 2}\right)
\end{aligned}\right.
$$


Medium 1 is considered as the cooling medium (heat flow), medium 2 is considered as the heating medium (cold flow), and $Q$ is heat transfer (heat load) of the heat exchanger.

\subsubsection{Temperature difference control algorithm}

Air compressor after cooler is mainly used to reduce the air inlet temperature of the air compressor behind the stage, as well as to reduce the power consumption of the compressor. Meanwhile, the heat transfer oil is used as medium to collect compression heat generated in the process of compression. The heat flow in the cooler after the air compressor is the air outlet of the air compressor (high temperature air $\rightarrow$ normal temperature air), and the cold flow will be heated (cold heat transfer oil $\rightarrow$ hot heat transfer oil). For the purpose of convenience, as shown in Fig. 6, the heat exchanger is simplified to a single tube structure, while cold flow uses the tube pass of the heat exchanger. The heat transfer length is $L$, the total heat transfer rate is $\Delta h$. At any point within the heat exchanger, the absolute value of enthalpy changes of both hot flow and cold flow are always equal, and the temperature of hot flow always higher than that of cold flow, $\Delta T_{\min }$ is the minimum heat transfer temperature difference between hot and cold flow in the heat exchanger.

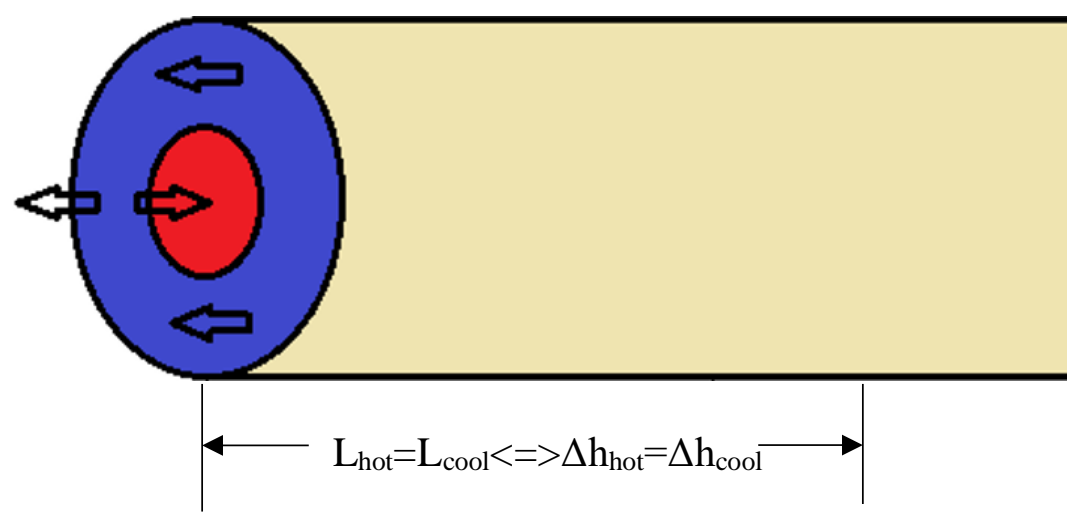

Fig. 6. Schematic diagram of simplified heat exchanger

It is recognized that different fluids have different physical properties and the characteristics of the temperature-enthalpy relationship is nonlinear, this will lead to the following phenomena in a counterflow heat exchanger: as enthalpy changes, the temperature of the two fluids changes in completely different directions. If the difference in internal temperature is not considered, 'temperature crossover' may occur, as shown in Fig.7. This is an important issue in designing the counterflow heat exchanger. In practice, the heat transfer will stop when the temperature of hot fluid and cold fluid is almost the same, and there will be no temperature crossover happened. 


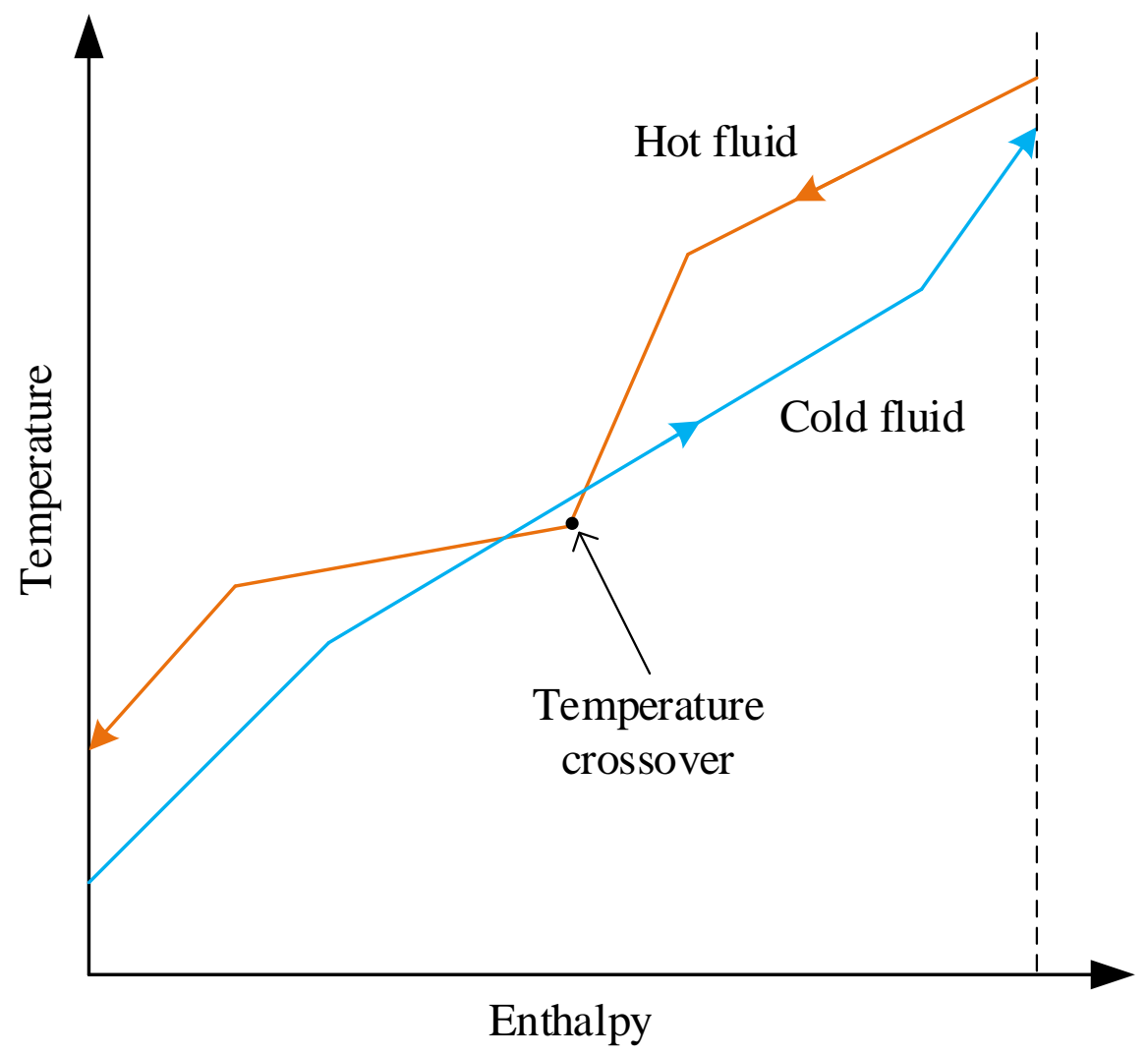

Fig. 7. An example of temperature crossover.

Referring to the temperature curves of heat transfer oil and air, it can be concluded that if the temperature difference at the low temperature end of the heat exchanger is reduced, the two temperature curves will inevitably cross. In order to obtain the hot heat transfer oil with high temperature, when calculating the rear cooler of the air compressor, the simulation platform provides a priority to ensuring that the outlet temperature of the cold flow (heat transfer oil) reaches the highest value, and then solves the optimal value of the other two optimized objective parameters. The specific algorithm is listed as follow:

(1) The hot end temperature of the air is obtained, and the temperature of cold heat transfer oil was selected.

(2) Assuming that the temperature difference at low temperature reaches the allowable minimum value of the heat exchanger, the corresponding heat transfer oil flow rate will be calculated, and the heat transfer oil flow rate reaches the theoretical maximum value.

(3) Under the condition of (1), the outlet temperature of the cold end of gets the minimum value, that is, both optimisation parameters can achieve the optimal value under this condition. However, due to the "temperature crossover" issue, this optimal condition is not valid, and the temperature difference at the low end must be amplified to avoid "temperature crossover".

(4) Starting from optimal value, take a small amount to gradually increase the outlet temperature of the cold end of the air. At this time, heat transfer of the cooler gradually decreases. Since the temperature of the heat transfer oil is fixed at the inlet of heat exchanger, the flow rate will gradually decrease.

(5) After obtaining the outlet temperature of a new air cold end, the new heat transfer oil flow rate is calculated. Afterwards, starting from the high temperature end of the cooler, the temperature curve of the cold and heat flow in the whole heat transfer process of the cooler is solved by small increment $d L$ or $d h$ to check whether the heat transfer conditions are satisfied. If yes, then the actual optimal values of the two optimisation parameters can be obtained simultaneously. If not, repeat step (4) and (5) until the heat transfer condition is satisfied.

Currently, the solution of the air compressor's rear cooler is completed, and all the temperature of the four end points are obtained, and the corresponding heat transfer oil flow rate is obtained. In the 
present work, pressure loss is considered in all heat exchangers, and the fluid pressure decreases linearly from inlet to outlet of heat exchanger. After solution is completed, the simulation platform CAESSC 2.0 can present the internal temperature curve of the heat exchanger and intuitively express to the user that there is no "temperature crossover" problem.

\subsubsection{Overall process}

As for A-CAES system, the energy storage/release processes are coupled since the compression heat produced during the compression stage will be used during the expansion process. Therefore, it is necessary that both processes are considered at the same time. According to the interstage heat transfer methods, there are three realizable multistage compression-expansion options:

(1) Adiabatic method: air is cooled only after the last stage of the compressor, and all of the compression heat is used to heat the air at the inlet of the first stage of the turbine;

(2) Semi-adiabatic method: air is cooled every two stages during compression and is heated every two stages during expansion;

(3) Polytropic method: air is cooled after each stage during compression, and heated before each stage during the expansion, as shown in Fig. 8, where mark 1 represents the atmosphere, mark 2 is CHTFST, mark 3 is the air storage system, and mark 4 represents HHTFST.

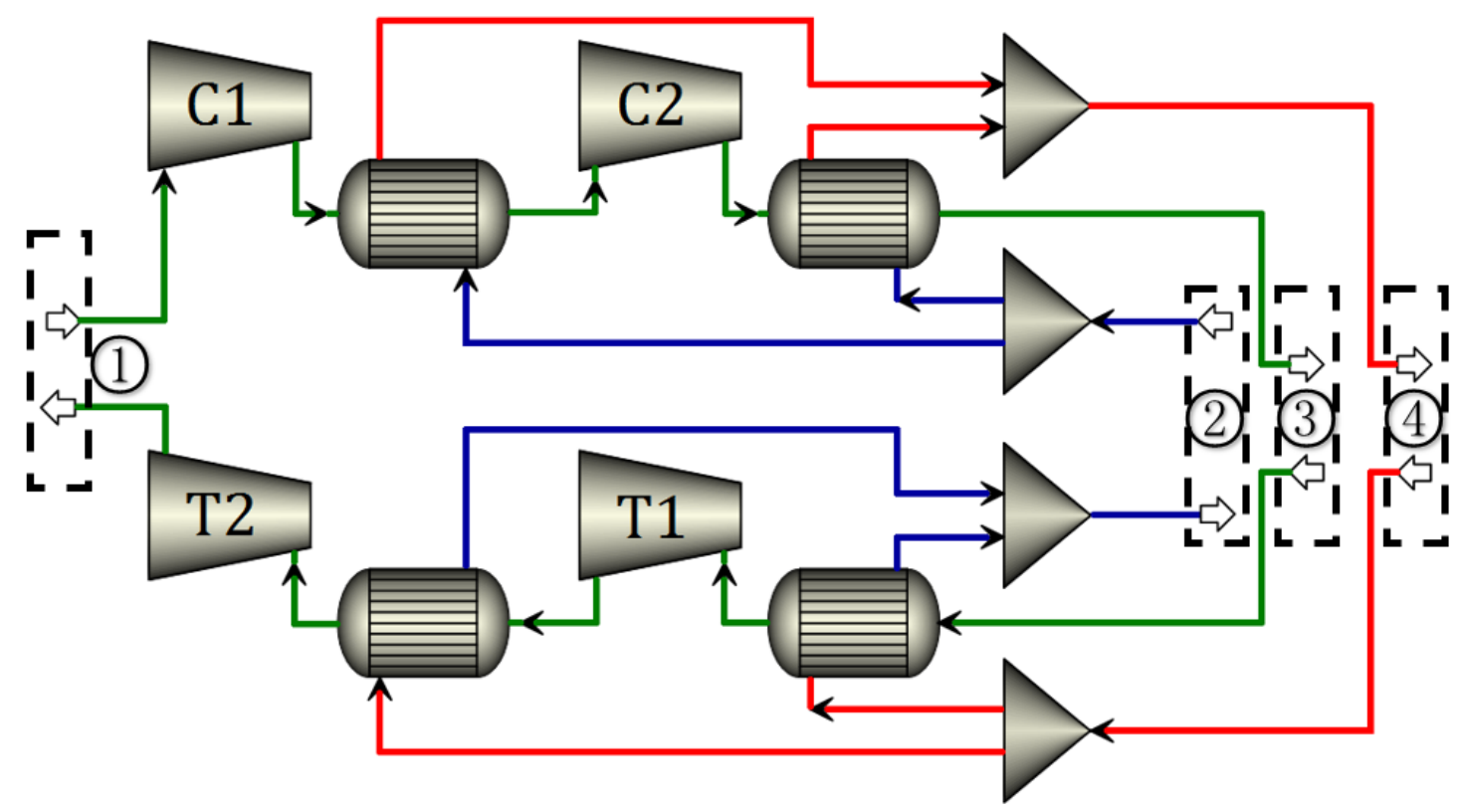

Fig. 8. Polytropic method of the system

In the current study, the efficiency of CAES system is defined as the ratio of net output energy to net input energy:

$$
\eta=\frac{W_{o u t}}{W_{i n}}=\frac{W_{e x} \eta_{g e}-W_{e l c_{-} 2}}{W_{c o} \eta_{m e}^{-1}+W_{e n c}+W_{e l c_{-} 1}}
$$

In the following derivations and calculations, $W_{e l c_{-} 1}$ and $W_{\text {elc } 2}$ are ignored. For A-CAES system, the polytropic method is applied, $W_{e n c}=0$. Assuming that all efficiencies of $n$ stages compressors are $\eta_{c o}$, and the efficiencies of $n$ stages turbines $\eta_{e x}$, then $\eta$ can be expressed as

$$
\eta=\frac{W_{e x} \eta_{g e}}{W_{c o} \eta_{m e}^{-1}}
$$

In the current study, assume air is ideal gas, with its constant specific heat, therefore,

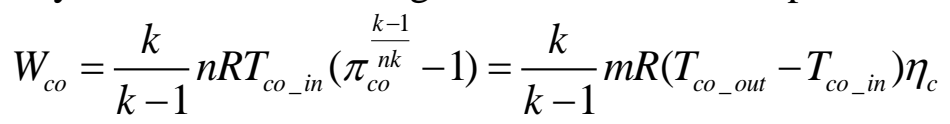


Ignore the loss of the heat exchangers, temperature differences in heat exchangers and loss of gas storage and heat storage, then

$$
T_{\text {ex_in }}=T_{\text {co_out }} \text { and } \pi_{c o}=\pi_{e x}=\pi
$$

According to the Eqs (6), (12), (20), (21) and (22), the $\eta$ can be given as

$$
\eta=\frac{n_{e x} \eta_{e x} \eta_{m e} \eta_{g e}\left(\pi^{\frac{k-1}{n_{c o} k}}+\eta_{c o}-1\right)\left(1-\pi^{\frac{k-1}{n_{e x} k}}\right)}{n_{c o}\left(\pi^{\frac{k-1}{n_{c o} k}}-1\right)}
$$

Usually $n_{c o}$ and $n_{e x}$ are equal to satisfy the condition of $T_{e x \_i n}=T_{c o_{-} o u t}$. Then Eq. (17) can be simplified into

$$
\eta=\frac{\eta_{e x} \eta_{m e} \eta_{g e}\left(\pi^{\frac{k-1}{n_{c o} k}}+\eta_{c o}-1\right)\left(1-\pi^{\frac{k-1}{n_{e x} k}}\right)}{\pi^{\frac{k-1}{n_{c o} k}}-1}
$$

when $n_{c o}=n_{e x}=1$, energy storage efficiency can reach the highest value, which is equivalent to the adiabatic method (actual stage number is not necessarily the 1). When $n_{e x} \rightarrow \infty, \pi^{\frac{k-1}{n_{c o} k}} \rightarrow 0$, then $\eta \rightarrow \eta_{c o} \eta_{e x} \eta_{m e} \eta_{g e}$, and this is the lowest limit for the efficiency in theory. This conclusion is drawn in terms of the polytropic method and can be generalized to three multistage compression-expansion methods, where $n_{c o}$ and $n_{e x}$ refer to the number of times of heat exchange during compressions and expansions respectively.

\subsection{Numerical model}

In the present work, an in-house code named CAESSC 2.0 (Compressed Air Energy Storage System Calculator) is developed to calculate the efficiencies of real A-CAES systems (considering various loss and using real air) with the above mentioned three methods. With the developed CAESSC 2.0, the numerical relations of temperature, pressure, enthalpy and entropy of real air are expressed with multiple nonlinear binary functions. The two main function models used in the program are:

$$
\begin{aligned}
& z=z_{0}+B x^{C}+D y^{E}+F x^{C} y^{E} \\
& z=z_{0}+A x+B y+C x^{2}+D x y+E y^{2}+F x^{3}+G x^{2} y+H x y^{2}+I y^{3}+J x^{4}+K x^{3} y+L x^{2} y^{2}+M x y^{3}+N y^{4}
\end{aligned}
$$

Fig. 9 shows the flow chart of the CAESSC 2.0. 


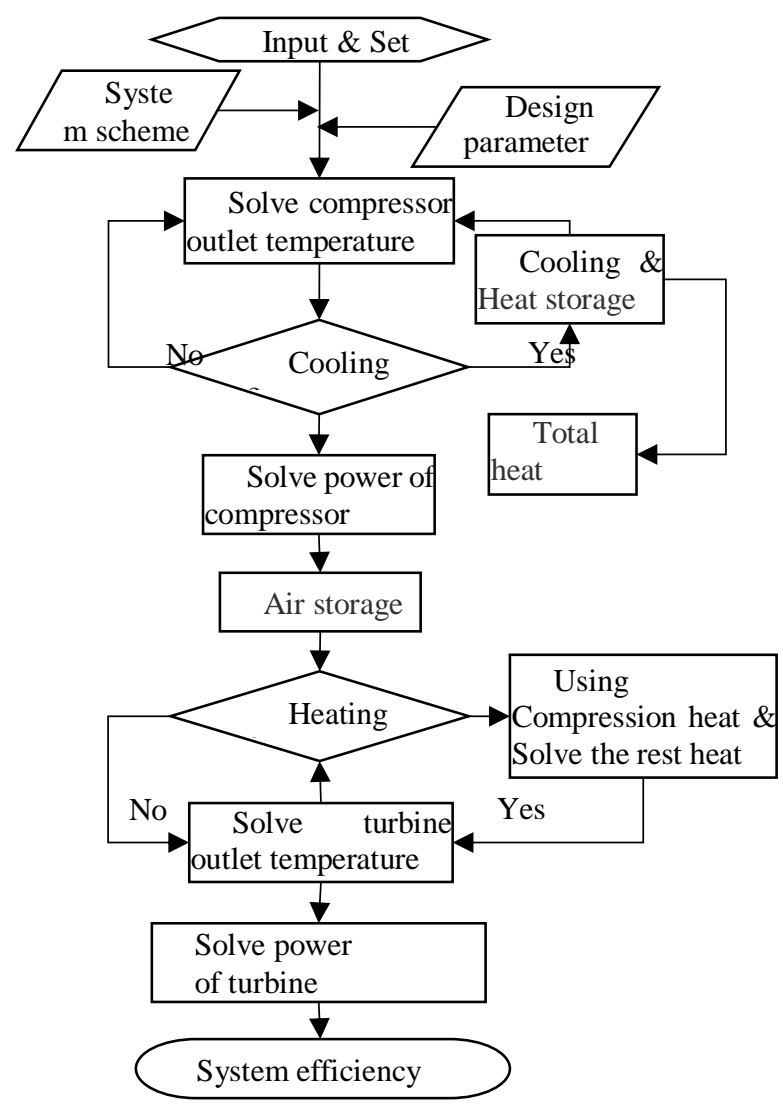

Fig. 9. Flow chart of analysis for the current A-CAES system.

\subsection{Operating parameters}

Before running CAESSC 2.0, operating parameters of the main component, including compressors, expanders and heat exchangers, should be defined. Table 1 lists the operating parameters for the current proposed system.

Table 1. Numerical simulation data for the proposed A-CAES system.

\begin{tabular}{|c|c|c|c|c|}
\hline $\begin{array}{l}\text { Type of } \\
\text { factors }\end{array}$ & Variable Name & Symbol & Unit & Variable value \\
\hline \multirow{2}{*}{$\begin{array}{l}\text { Inlet } \\
\text { conditions }\end{array}$} & Inlet total temperature & $\mathrm{T}_{\text {cin } 1}$ & $\mathrm{~K}$ & 288.15 \\
\hline & Inlet total pressure & $\mathrm{p}_{\mathrm{cin} 1}$ & $\mathrm{MPa}$ & 0.101 \\
\hline \multirow{3}{*}{$\begin{array}{l}\text { System } \\
\text { configuration }\end{array}$} & $\begin{array}{l}\text { No. of compression stages } \\
\text { No. of air coolers }\end{array}$ & & & $\begin{array}{l}6 \\
1 \text { or } 3 \text { or } 6\end{array}$ \\
\hline & No. of turbine stages & & & 4 \\
\hline & No. of air heaters & & & 1 or 3 or 4 \\
\hline \multirow{11}{*}{$\begin{array}{l}\text { System } \\
\text { Component } \\
\text { parameters }\end{array}$} & Isentropic efficiency of compressors & & & $\begin{array}{l}0.855,0.85,0.85 \\
0.85,0.84 .0 .83\end{array}$ \\
\hline & Isentropic efficiency of turbines & & & $\begin{array}{l}0.870,0.875 \\
0.890,0.885\end{array}$ \\
\hline & Rotor mechanical efficiency & & & 0.99 \\
\hline & motor efficiency & & & 0.985 \\
\hline & generator efficiency & & & 0.98 \\
\hline & increasing gearbox efficiency & & & 0.97 \\
\hline & reduction gearbox efficiency & & & 0.97 \\
\hline & Pressure drop of coolers & $\mathrm{dp}$ & $\mathrm{kPa}$ & 10 \\
\hline & Heat storage efficiency of HHTFST & & & 0.985 \\
\hline & Gas storage efficiency & & & 0.99 \\
\hline & Heat transfer temperature difference & $\mathrm{dT}$ & $\mathrm{K}$ & 10 \\
\hline
\end{tabular}




\section{Results and discussion}

3.1. Optimisation results of heat exchanger in A-CAES system

In CAESSC 2.0, the heat transfer oil was used as the thermal medium. Under the same working conditions, the internal temperature curve of the heat exchanger obtained by this program was compared with ASPEN PLUS, a commercial software. Results show that they are in a decent agreement, as shown in Fig.10 to Fig.13. The x-axis is the heat load ratio that is defined by the enthalpy difference to the total heat transfer capacity.

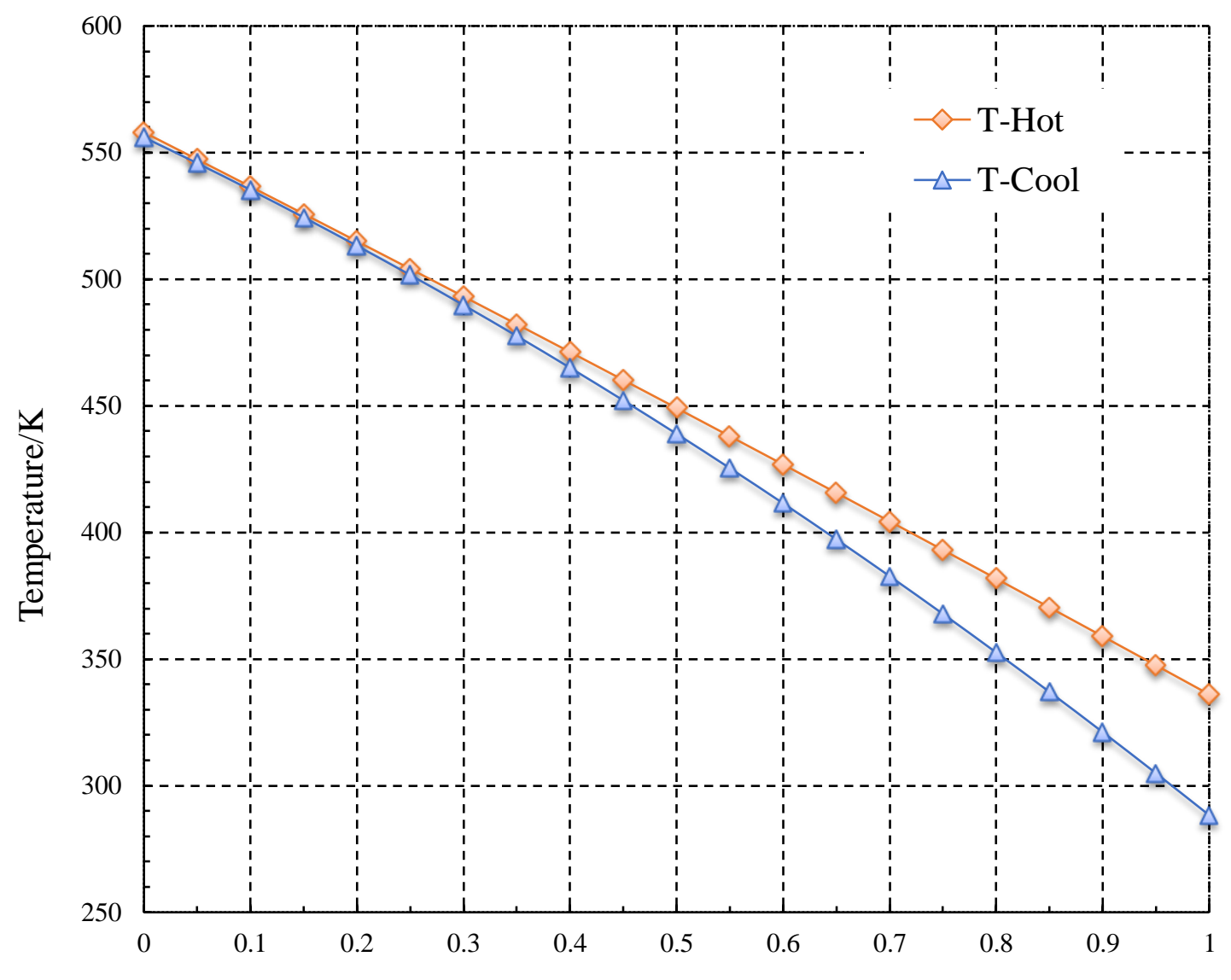

Heat load ratio

Fig. 10. Internal temperature curve of the rear cooler of the main compressor unit (CAESSC 2.0). 


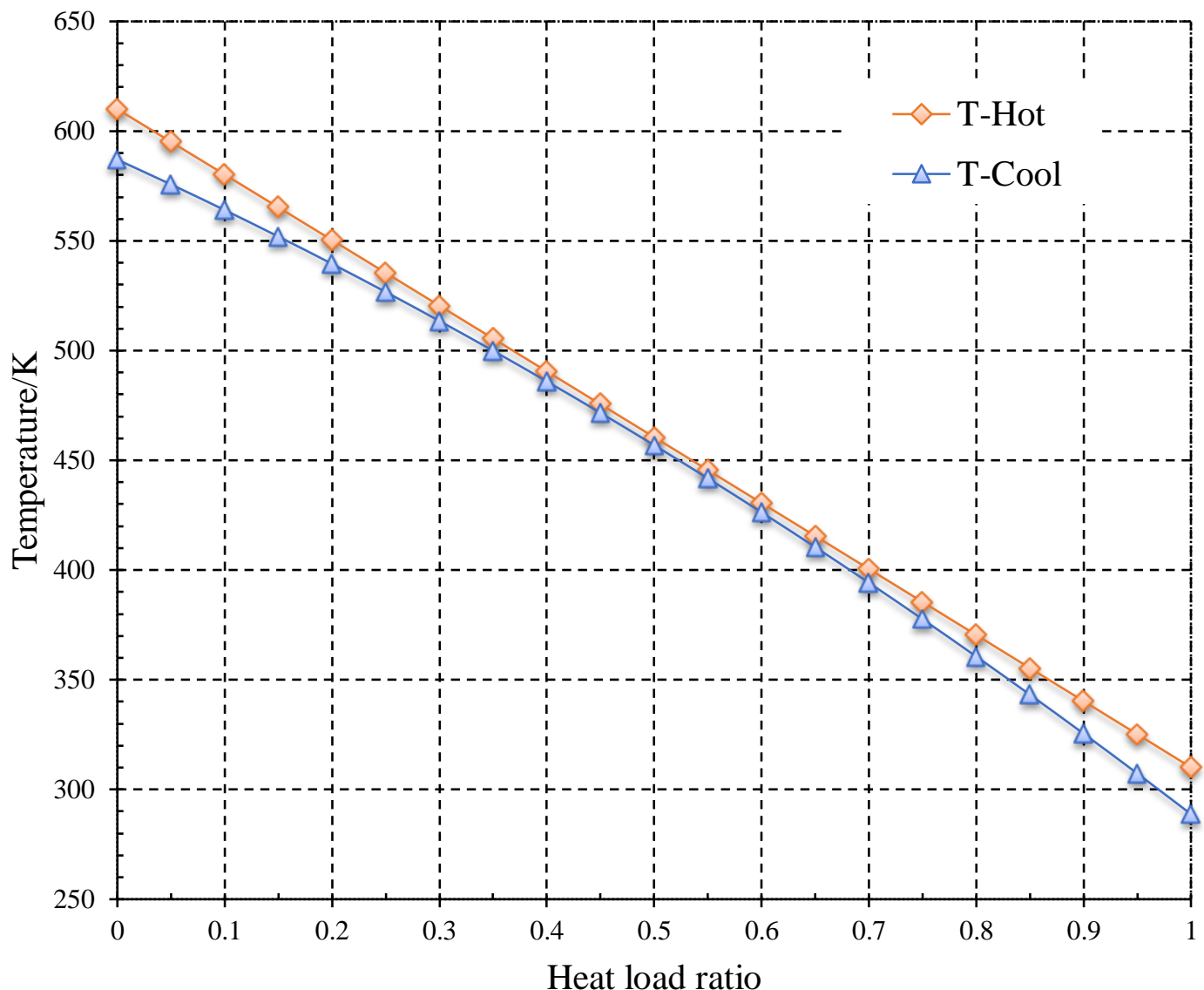

Fig. 11. Internal temperature curve of aftercooler of circulating compressor unit (CAESSC 2.0).

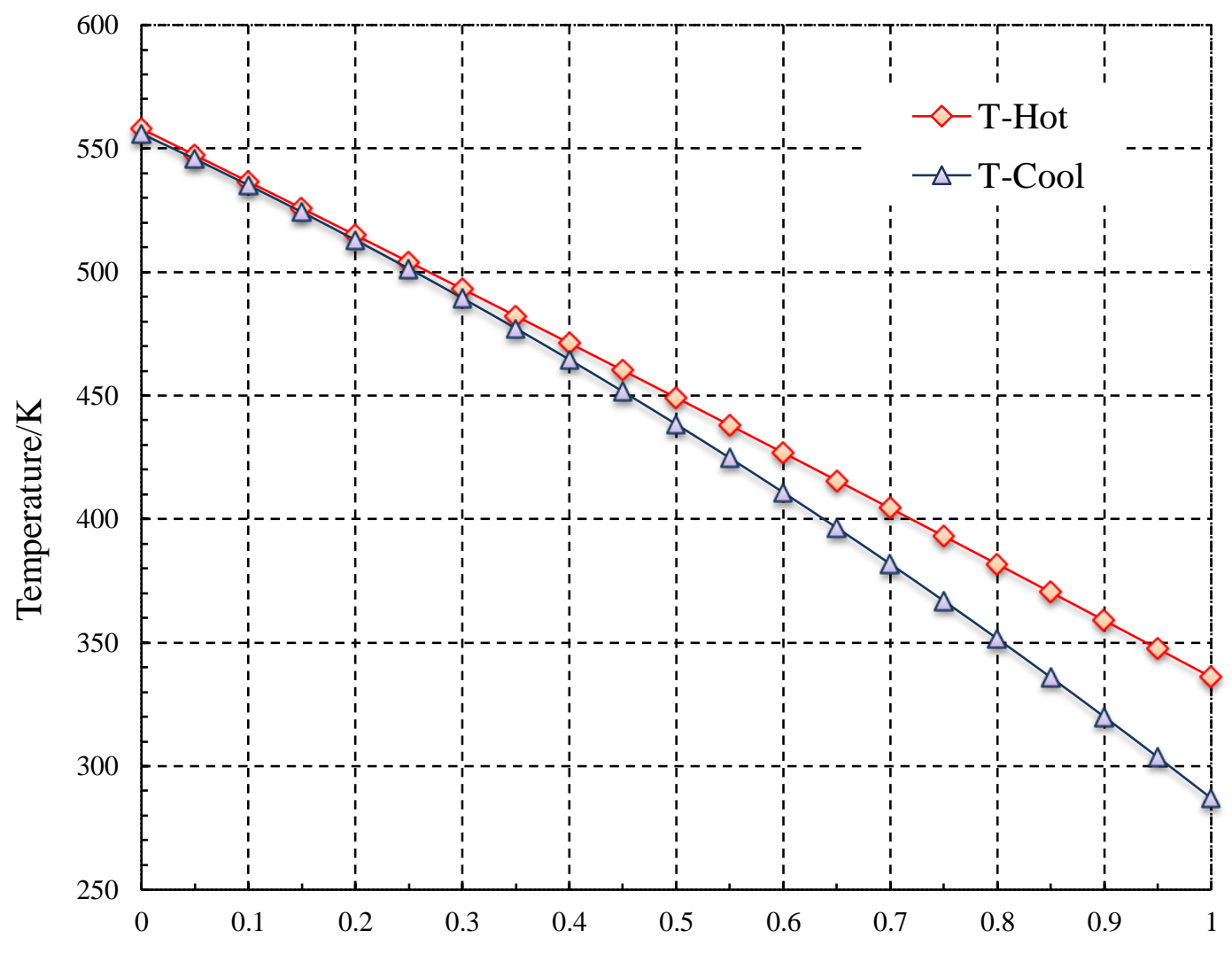

Heat load ratio

Fig. 12. Internal temperature curve of the rear cooler of the main compressor unit (Aspen Plus). 


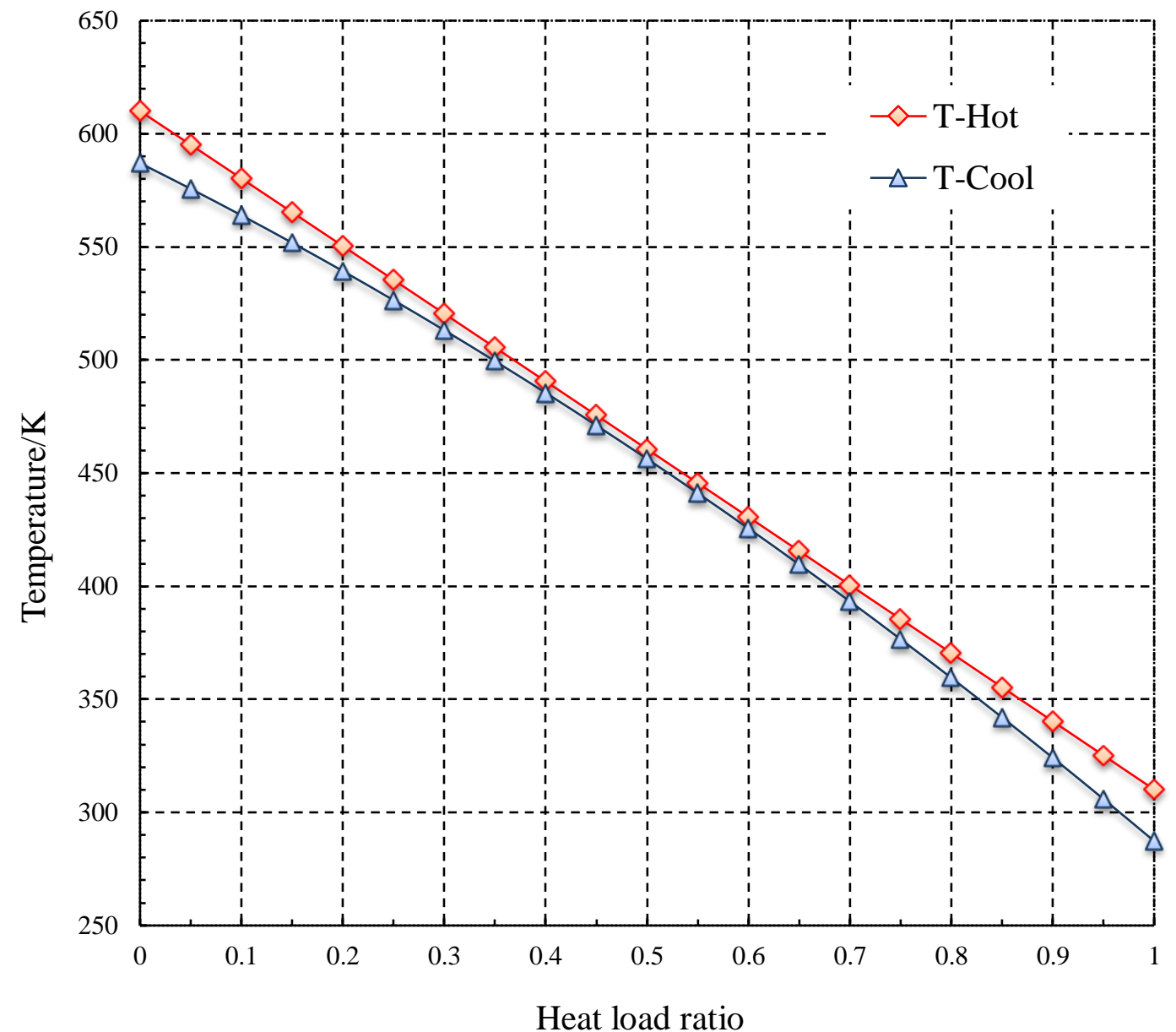

Fig. 13. internal temperature curve of heat exchanger after circulating compressor unit (Aspen Plus).

The influence of inlet temperature of turbine on overall system efficiency is investigated under the same conditions. From Fig. 14, the calculation results obtained from CAESSC 2.0 agree well with the data calculated with commercial software.

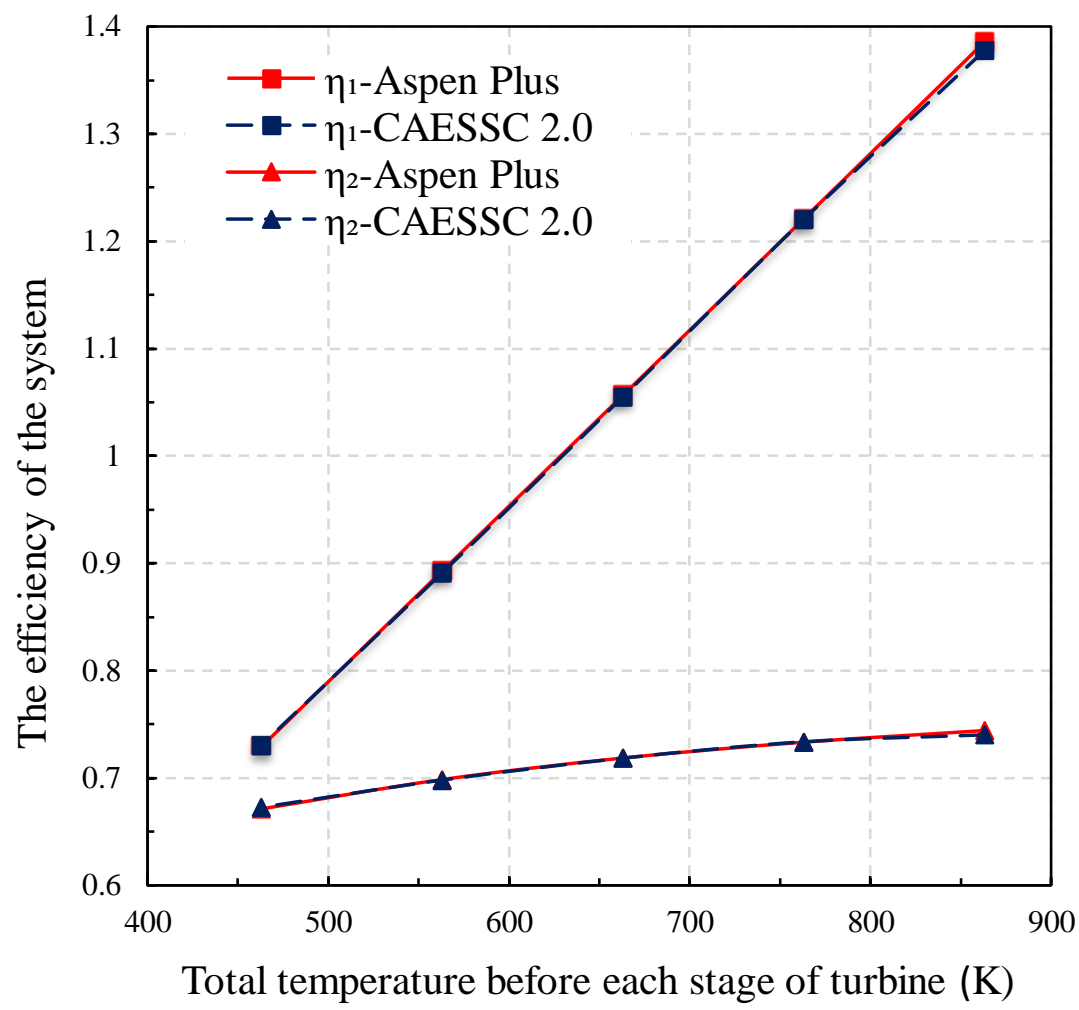

Fig. 14. Influence of inlet temperature on system efficiency 


\subsection{Effect of gas storage methods on energy storage density}

According to the gas storage methods, CAES systems are mainly divided into two methods: constant volume and constant pressure. Working under the constant volume method, the inlet pressure of the turbine $P_{i n}$ is lower than the gas storage pressure $P_{s t o}$, therefore throttling is usually used before the inlet of turbine. Throttling pressure ratio (TPR) is defined as the ratio of $P_{\text {sto }}$ to $P_{\text {inn }}$. Both commercial power stations, Huntorf and McIntosh, use the constant volume method, and their throttling pressure ratios are 1.4 and 1.6 , respectively.

Assuming the total temperatures of air before and after the throttle are always equal to the ambient temperature ( $\left.T_{\mathrm{out}}=T_{\mathrm{in}}=T_{\mathrm{amb}}\right)$. For ideal gas, the expression of the available energy loss during the throttling process is as follows

$$
e_{L}=R T_{a m b} \ln \frac{P_{s t o}}{P_{i n}}
$$

And the available energy of the compressed gas $\left(P_{1}, T_{0}\right)$ relative to environment $\left(P_{a m b}, T_{a m b}\right)$ is

$$
e_{1}=R T_{a m b} \ln \frac{P_{s t o}}{P_{a m b}}
$$

According to Eqs. (27) and (28), the proportion of gas available energy loss that caused by throttling $\left(e_{L} / e_{1}\right)$ under different gas pressures and throttle pressure ratios can be obtained, as shown in Fig. 15. It can be seen clearly that the highest available energy loss can be up to 0.48 with throttling pressure ration of 3.0 at $1 \mathrm{MPa}$. Under different TPR values, the available energy loss presented significant decreases with the increase of gas storage pressure at the initial stage (from 1 to $10 \mathrm{MPa}$ ). There are approximate liner drops displayed after storage pressure rise from $10 \mathrm{MPa}$ to $30 \mathrm{MPa}$. Less TPR value leaded to less available energy loss under a constant storage pressure.

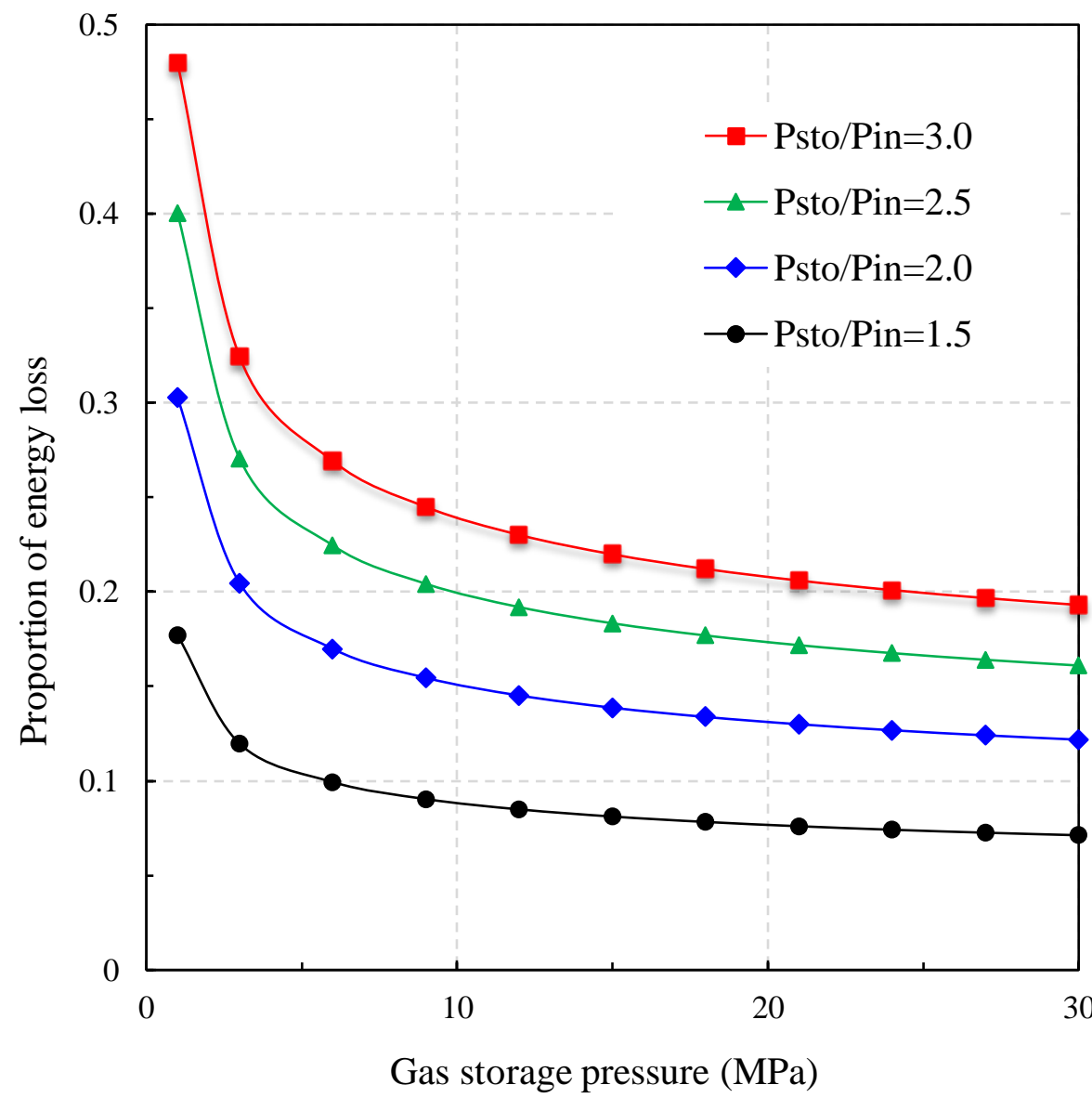

Fig.15. The percentage of available energy loss caused by throttling. 
Thus, in order to obtain constant pressure before the inlet of the turbine under constant volume method, large available energy loss is inevitable. And the loss increases with the bigger and bigger TPR. However, smaller TPR would cause lower energy storage density.

According to the actual physical property parameters, the relative energy storage density (the ratio of energy storage density under constant volume method to constant pressure method) is obtained under different gas pressures and throttling pressure ratios, as shown in Fig.16. Relative energy storage density is kept constant with the gas storage pressure ranging from $1 \mathrm{MPa}$ to $10 \mathrm{MPa}$. Afterwards, it starts to drop when the pressure is over $10 \mathrm{MPa}$. According to the above, the design of constant pressure gas storage system is of great significance to improve the overall performance of the compressed air energy storage system.

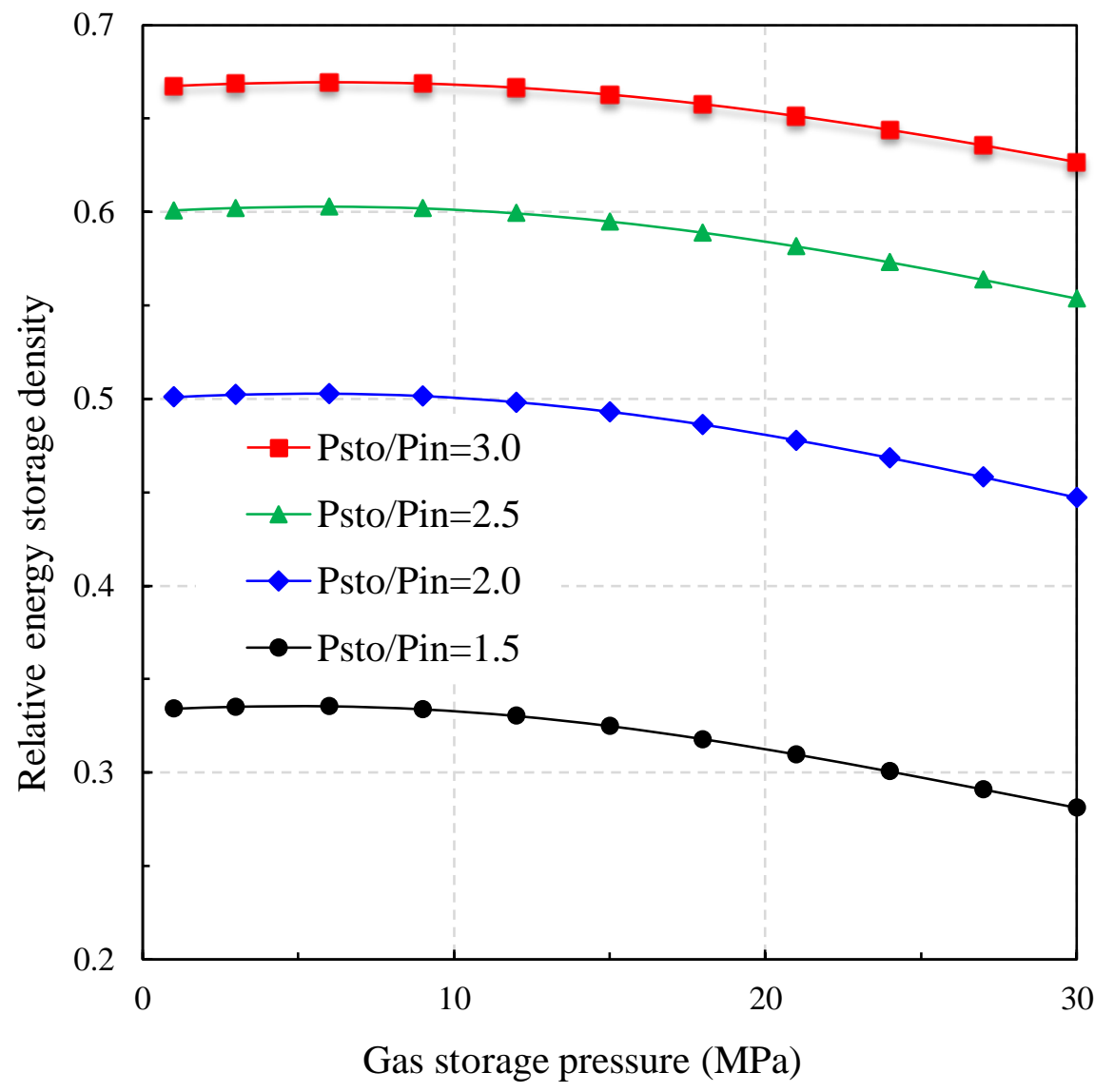

Fig.16. The relative energy storage density under the constant volume method.

For the CAES system using deep mine to store gas, connecting the mine cavern with a pool on the ground, it would keep the storage air with the constant pressure, as shown in Fig.1 (the gas storage pressure depends on the depth of the mine).

\subsection{Effect of heat transfer mode on overall efficiency}

The overall efficiencies of A-CAES system with three different heat transfer modes are calculated and compared, as shown in Fig. 17. Results show that the overall efficiency can reach the highest value of $71 \%$ when adopting the adiabatic method, although the method is the most uneconomical for the compression process, whereas the efficiency for isothermal process is around 58\%. This conclusion consists with the theoretical analysis that discussed previously. Since a large amount of high-grade compression heat produced during the adiabatic compression process, the heat could be fully used during the energy release process, thus the output power of the turbine is greatly increased relatively. Under the same operating conditions where the throttling pressure ratio is 1.43 (turbine inlet pressure is $70 \%$ of the storage pressure), the system efficiency of the CAES with constant gas 
storage pressure can reach to $71 \%$, this shows $4 \%$ higher than that using constant volume gas storage. Due to a constant pressure, both expansion ratio and energy storage density can be achieved for the exhaust gas.

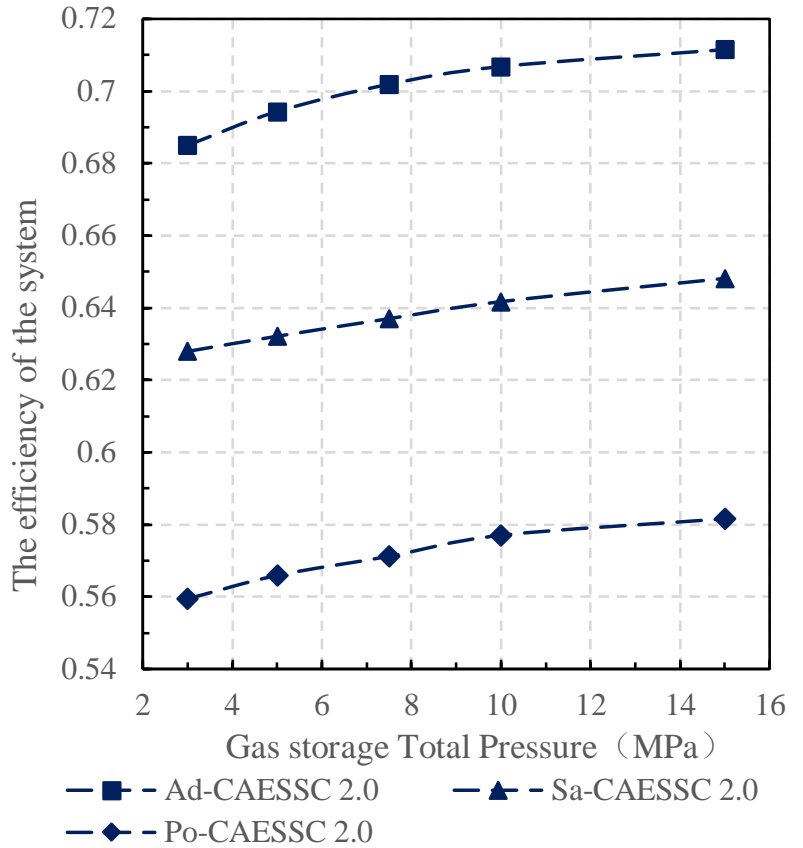

(a) Constant pressure gas

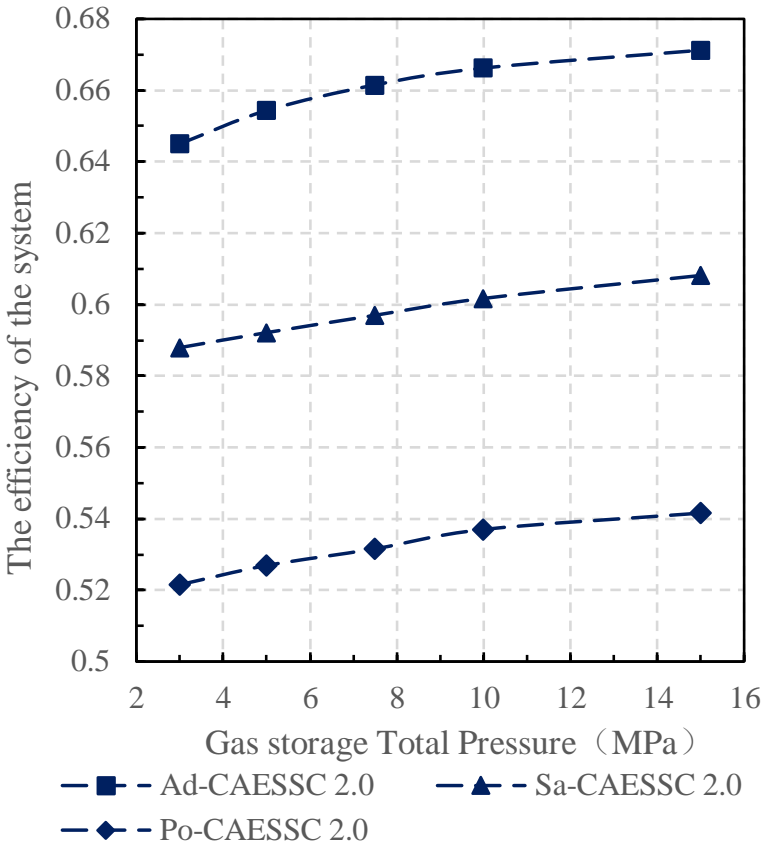

(b) Constant volume is deflated

Fig. 17. Overall energy storage efficiencies of adiabatic CAES systems with the three compression-expansion methods.

\section{Conclusions}

(Ad- Adiabatic method, Sa- Semi-adiabatic method, Po- Polytropic method)

The current work investigates the theoretical potential of a new A-CAES system through an inhouse code named CAESSC 2.0, and discusses the importance of several process variables such as heat transfer mode, stage number of compressor and turbine. Based on the results of the numerical studies, the following specific conclusions may be achieved:

(1) The efficiencies of the real A-CAES system under different configurations were calculated with current code and validated against reference data and commercial software. It shows that the program has the same accuracy and higher computational efficiency as the commercial simulation software.

(2) The highest efficiency could be obtained with the adiabatic method for the adiabatic CAES system. The overall efficiency of adiabatic compressed air energy storage system can exceed $70 \%$ when using compressed air as thermal conductivity.

(3) Using the constant pressure method of gas storage can improve the energy storage efficiency and the energy storage density of the system significantly. Under the same condition, the efficiency of the system under constant pressure storage is about $4 \%$ higher than that under constant volume storage.

\section{Acknowledgments}

The authors would like to thank the financial support from State Grid Coporation of China Research Program "Preliminary Study of Frequency Modulation Technology for Power Grid Based on Compressed Air Energy Storage" (SGGR0000DLJS1800287).

\section{References}

[1] Weitemeyer, S., Kleinhans, D., Vogt., T., Agert, C. Integration of renewable energy sources in 
future power systems: the role of storage. Renew Energy 2015; 75:14-20.

[2] Luo, X., Wang, J., Dooner, M., Clarke, J. Overview of current development in electrical energy storage technologies and the application potential in power system operation. Applied Energy 2015; 137:511-536.

[3] Wu, S., Zhou, C., Doroodchi, E., Moghtaderi, B. Thermodynamic analysis of a novel hybrid thermochemical-compressed air energy storage powered by wind, solar and/or off-peak electricity. Energy Conversion and Management 2019; 180:1268-1280.

[4] Liu, S., Wu, S., Hu, Y., Li, H. Comparative analysis of air and CO2 as working fluids for compressed and liquefied gas energy storage technologies. Energy Conversion and Management 2019; 181:608-620.

[5] Luo, X., Wang, J., Dooner, M., Clarke, J., Krupke, C. Modelling study, efficiency analysis and optimisation of large-scale adiabatic compressed air energy storage systems with lowtempearture thermal storage. Applied Energy 2016; 162:589-600.

[6] Crotogino, F., Mohmeyer, K., Scharf, R.. Huntorf CAES: more than 20 Years of successful operation. Orlando, Florida, USA: Spring 2001 Meeting; 2001; 1-6.

[7] Safaei, H., Keith, D.W., Hugo, R.J. Compressed air energy storage (CAES) with compressors distributed at heat loads to enable waste heat utilization. Applied Energy 2013;103:165-179.

[8] Smith, D. The first CAES merchant. Mod Power System 2001.

[9] Guo, C., Xu, Y., Zhang, X., Guo, H., Zhou, X., Liu, C., Qin, W., Li, W., Dou, B., Chen, X. Performance analysis of compressed air energy storage systems considering dynamic characteristics of compressed air storage. Energy 2017; 135:876-888.

[10] He, W., Luo, X., Evans, D., Busby, J., Garvey, S., Parkes, D., Wang, J. Exergy storage of compressed air in cavern and cavern volume estimation of the large-scale compressed air energy storage system. Applied Energy 2017; 208:745-757.

[11] Cheayb, M., Gallego, M.M., Tazerout, M., Poncet, S. Modelling and experimental validationof a small-scale trigenerative compressed air energy storage system 2019; 239:1371-1384.

[12] Zhang, S., Wang, H., Li, R., Li, C., Hou, F., Ben, Y. Thermodynamic analysis of cavern and throttle value in large-scale compressed air energy storage system. Energy Conversion and Management 2019; 183:721-731.

[13] Al Jubori, A.M., Jawad, Q.A. Investigation on performance improvement of small scale compressed-air energy storage system based on efficient radial-inflow expander configuration. Energy Conversion and Management 2019; 182:224-239.

[14] Guo, C., Xu, Y., Guo, H., Zhang, X., Lin, X., Wang, L., Zhang, Y., Chen, H. Comprehensive exergy analysis of the dynamic process of compressed air energy storage system with lowtemperature thermal energy storage. Applied Thermal Engineering 2019; 147:684-693.

[15] He, Q., Li, G., Lu, C., Du, D., Liu, W. A compressed air energy storage system with variable pressure ratio and its operation control. Energy 2019; 169:881-894.

[16] Zhang, Y., Yang, K., Li, X., Xu, J. The thermodynamic effect of air storage chamber model on advanced adiabatic compressed air energy storage system. Renewable Energy 2013;57:469-478.

[17] Yang, K., Zhang, Y., Li, X., Xu, J. Theoretical evaluation on the impact of heat exchanger in advanced adiabatic compressed air energy storage system. Energy Conversion and Management 2014; 86:1031-1044.

[18] Barbour, E., Mignard, D., Ding, Y., Li, Y. Adiabatic compressed air energy storage with packed bed thermal energy storage. Applied Energy 2015; 155:804-815.

[19] Mozayeni, H., Negnevitsky, M., Wang, X., Cao, F., Peng, X. Performance study of an advanced adiabatic compressed air energy storage system. Energy Procedia 2017; 110:71-76.

[20] Geissbuhler, L., Becattini, V., Zanganeh, G., Zavattoni, S., Barbato, M., Haselbacher, A. Pilotscale demonstration of advanced adiabatic compressed air energy storage, Part 1: Plant description and tests with sensible thermal-energy storage. Journal of Energy Storage 2018; 17:129-139.

[21] Becattini, V., Geissbuhler, L., Zanganeh, G., Haselbacher, A., Steinfeld, A. Pilot-scale demonstration of advanced adiabatic compressed air energy storage, Part 2: Tests with 
combined sensible/latent thermal-energy storage. Journal of Energy Storage 2018; 17:140-152.

[22] Han, Z., Guo, S. Investigation of discharge characteristics of a tri-generative system based on advanced adiabatic compressed air energy storage. Energy Conversion and Management 2018; 176:110-122.

[23] Yang, L., Cai, Z., Li, C., He, Q., Ma, Y., Guo, C. Numerical investigation of cycle performance in compressed air energy storage in aquifers. Applied Energy 2020; 269: 115044.

[24] Meng, J., Wei, M., Song, P., Tian, R., Hao, L., Zheng, S. Performance evaluation of a solar transcritical carbon dioxide Rankine cycle integrated with compressed air energy storage. Energy Conversion and Management 2020; 215: 112931.

[25] Mohamad, C., Mylène, M., Sébastien, P., Mohanda, T. Micro-scale trigenerative compressed air energy storage system: Modeling and parametric optimization study. Journal of Energy Storage 2019. 26: 100944.

[26] Chen, S., Arabkoohsar, A., Zhu, T., Nielsen, M. Development of a micro-compressed air energy storage system model based on experiments. Energy 2020; 195: 117152. 